\title{
Time-resolved dual transcriptomics reveal early induced Nicotiana benthamiana root genes and conserved infection-promoting Phytophthora palmivora effectors
}

Edouard Evangelisti ${ }^{1 \dagger}$, Anna Gogleva ${ }^{1 \dagger}$, Thomas Hainaux $^{1,2}$, Mehdi Doumane ${ }^{1,3}$, Frej Tulin', Clément Quan ${ }^{1}$, Temur Yunusov ${ }^{1}$, Kévin Floch ${ }^{1}$ and Sebastian Schornack ${ }^{1 *}$ (D)

\begin{abstract}
Background: Plant-pathogenic oomycetes are responsible for economically important losses in crops worldwide. Phytophthora palmivora, a tropical relative of the potato late blight pathogen, causes rotting diseases in many tropical crops including papaya, cocoa, oil palm, black pepper, rubber, coconut, durian, mango, cassava and citrus. Transcriptomics have helped to identify repertoires of host-translocated microbial effector proteins which counteract defenses and reprogram the host in support of infection. As such, these studies have helped in understanding how pathogens cause diseases. Despite the importance of $P$. palmivora diseases, genetic resources to allow for disease resistance breeding and identification of microbial effectors are scarce.

Results: We employed the model plant Nicotiana benthamiana to study the P. palmivora root infections at the cellular and molecular levels. Time-resolved dual transcriptomics revealed different pathogen and host transcriptome dynamics. De novo assembly of P. palmivora transcriptome and semi-automated prediction and annotation of the secretome enabled robust identification of conserved infection-promoting effectors. We show that one of them, REX3, suppresses plant secretion processes. In a survey for early transcriptionally activated plant genes we identified a $N$. benthamiana gene specifically induced at infected root tips that encodes a peptide with danger-associated molecular features.
\end{abstract}

Conclusions: These results constitute a major advance in our understanding of $P$. palmivora diseases and establish extensive resources for $P$. palmivora pathogenomics, effector-aided resistance breeding and the generation of induced resistance to Phytophthora root infections. Furthermore, our approach to find infection-relevant secreted genes is transferable to other pathogen-host interactions and not restricted to plants.

Keywords: Dual transcriptomics, Effectors, RXLR effectors, Secretome, De novo transcriptome assembly, N. benthamiana, P. palmivora, Non-model species

\footnotetext{
* Correspondence: sebastian.schornack@slcu.cam.ac.uk

${ }^{\dagger}$ Equal contributors

'Sainsbury Laboratory Cambridge University (SLCU), Cambridge, UK

Full list of author information is available at the end of the article
} 


\section{Background}

Phytophthora is a genus of plant-pathogenic oomycetes responsible for economically important losses in crops worldwide, as well as damage to natural ecosystems [1]. Phytophthora infestans is the causal agent of tomato and potato late blight in temperate climates and contributed to major crop losses during the Great Irish Famine [2]. Phytophthora palmivora, a tropical relative of $P$. infestans originating from southeastern Asia [3] but now present worldwide due to international trade [4], causes root, bud and fruit rotting diseases in many important tropical crops such as papaya, cocoa, oil palm, black pepper, rubber, coconut, durian, mango, cassava and citrus [5-8]. In addition, $P$. palmivora infects the roots and leaves of several model plant species such as Medicago truncatula [9], Hordeum vulgare [10] and Arabidopsis thaliana [11]. Despite its economic impact and widespread distribution, nothing is known about the molecular basis underlying its ability to infect its host species and the root responses associated with an infection.

P. palmivora has a hemibiotrophic lifestyle. Similar to other Phytophthora species, its asexual life cycle in plants is characterised by adhesion of mobile zoospores to the host tissue, encystment and germ tube formation [12]. Entry into the plant is achieved via surface appressoria and is followed by establishment of an apoplastic hyphal network. During this biotrophic stage, P. palmivora projects haustoria into plant cells. These contribute to acquisition of nutrients and release virulence proteins known as effectors [13]. This is followed by a necrotrophic stage characterised by host tissue necrosis and the production of numerous sporangia which release zoospores [14].

Sequencing of Phytophthora genomes and transcriptomes has revealed repertoires of effector proteins that counteract plant defenses and reprogram the host in support of infection. Secretome predictions and subsequent evolutionary and functional studies have helped us to understand how these pathogens cause diseases $[15,16]$. Oomycete effectors are secreted into the apoplast of infected plants. Some of them act inside plant cells, and conserved RXLR or LFLAK amino acid motifs in their N-terminal parts have been associated with their translocation from the microbe into the host cell $[17,18]$. The LFLAK motif is present in Crinkler (CRN) effectors, named after a crinkling and necrosis phenotype caused by some CRN proteins when expressed in plants [19]. RXLR effectors are usually short proteins with little similarity to conserved functional domains in their $\mathrm{C}$ termini. They localise to diverse subcellular compartments and associate with plant target proteins with key roles during infection [20].

Recent studies on bacterial and oomycete plant pathogens identified subsets of effectors that are conserved amongst a large number of strains. These so-called core effectors are responsible for a substantial contribution to virulence and thus cannot be mutated or lost by the pathogen without a significant decrease in virulence [21]. Thus, core effectors constitute highly valuable targets for identification of resistant germplasm and subsequent breeding of disease-resistant crops [21-23]. To date, the occurrence of such core effectors in oomycetes has largely been reported from plant pathogens with a narrow economical host range such as Hyaloperonospora arabidopsidis, Phytophthora sojae [24] and P. infestans [25].

Plants have evolved a cell-autonomous surveillance system to defend themselves against invading microbes [26]. Surface-exposed pattern recognition receptors (PRRs) recognise conserved microbe-associated molecular patterns (MAMPs) released during infection, such as the Phytophthora transglutaminase peptide Pep-13 [27, 28]. Plants are also able to recognise self-derived damage-associated molecular patterns (DAMPs). These include intracellular peptides that are released in the apoplast upon wounding, such as systemins [29] and secreted plant peptide precursors with DAMP features that are processed in the apoplast [30-32]. Pathogen recognition initiates basal defense responses which include activation of structural and biochemical barriers, known as MAMP-triggered immunity (MTI) [26]. Plant pathogens are able to overcome MTI by secreting effectors that suppress or compromise MTI responses, thereby facilitating effector-triggered susceptibility (ETS). In response, plants have evolved disease resistance proteins to detect pathogen effectors or effector-mediated modification of host processes, leading to effectortriggered immunity (ETI) [26]. Phytophthora genes encoding effectors which trigger a resistance response in host plants carrying the cognate disease resistance gene are often termed avirulence (AVR) genes. Cross-species transfer of PRRs and disease resistance genes against conserved MAMPs or AVR proteins has been successfully employed to engineer resistant crops [33, 34].

Host cell responses to oomycete infections have mainly been studied in aboveground tissues and notably involve subcellular rearrangements of the infected cells, including remodelling of the cytoskeleton $[14,35,36]$ and focal accumulation of secretory vesicles [37, 38], which contribute to defense by delivering antimicrobial compounds to the extrahaustorial matrix $[39,40]$. Endocytic vesicles accumulate around oomycete haustoria [41], and the plant-specific small GTPase RAB5 is recruited at the extrahaustorial membrane during Arabidopsis infection by obligate biotrophs [42]. Several oomycete effectors target different stages of the host secretory pathway. In the apoplast, pathogen-secreted inhibitors have been associated with defense suppression. For instance, the apoplastic effector GIP1 from $P$. sojae inhibits the soybean endoglucanase EGaseA [43]. The $P$. infestans Kazal-like protease inhibitors EPI1 [44] 
and EPI10 [45] inhibit the Solanum lycopersicum defense protease $\mathrm{P} 69 \mathrm{~B}$. The cystatin-like protease inhibitors EPIC1 and EPIC2B inhibit the cysteine proteases PIP1 (Phytophthora-inhibited protease 1) [46] and Rcr3 [47] as well as the papain-like protease C14 [48]. Interestingly, expression of the $P$. infestans RXLR effector AVRblb2 in plant cells prevents C14 protease secretion and causes an accumulation of protease-loaded secretory vesicles around haustoria [49].

In this study, we employ the model plant Nicotiana benthamiana [50] to study root infection by P. palmivora. Dual transcriptomics and de novo assembly of the P. palmivora transcriptome allowed us to define pathogen and plant genes expressed during the interaction. We identified major shifts in pathogen gene expression dynamics associated with lifestyle changes which, interestingly, are not mirrored by dramatic shifts in plant gene expression patterns. De novo transcriptome assembly coupled with semi-automatic secretome prediction and annotation allowed us to identify a set of more than 2000 secreted Phytophthora proteins. This approach enabled discovery of an abundant class of potential novel effectors carrying a conserved peptide motif (HxGPCExxxDD) and also allowed us to pinpoint key effectors' contribution to the $P$. palmivora infection strategy. We characterised two conserved RXLR effectors, REX2 and REX3, that promote root infection upon expression in plants. We furthermore show that REX3 was able to interfere with host secretion. By studying host transcriptional changes upon infection, we identified a gene encoding a secreted peptide precursor with potential DAMP motifs whose promoter was specifically activated at root tip infection sites. Hence, our work establishes a major resource for root-pathogen interactions, showcases examples of how to exploit these data and provides inroads for effector-aided resistance breeding in tropical crops.

\section{Results}

Phytophthora palmivora exerts a hemibiotrophic lifestyle in Nicotiana benthamiana roots

To describe the infection development of the root pathogen $P$. palmivora, we investigated the infection dynamics of hydroponically grown $N$. benthamiana plants root-inoculated with P. palmivora LILI-YKDEL [9] zoospores (Fig. 1). Disease development was classified into different symptom extent stages (SESs) which allowed us to follow the systemic spread of the infection from roots to shoots (Fig. 1a). The plants looked healthy for up to 3 days (symptom extent stage 1, SES 1). Disease progression in the aerial parts then resulted in a shrunken, brown hypocotyl and wilting of the oldest leaves (SES 2). This was rapidly followed by brown coloration and tissue shrinkage of the stem (SES 3) up to the apex (SES 4). Infected plants eventually died within 8 to 10 days
(SES 5), indicating that $N$. benthamiana is susceptible to root infection by P. palmivora (Fig. 1a).

We next characterised the P. palmivora- $N$. benthamiana interaction on the microscopic level using the fluorescently labelled isolate LILI-YKDEL (Fig. 1b-h). Infection events were observed at $3 \mathrm{~h}$ after inoculation (hai). Zoospores were primarily attracted to root tips, where they encysted and germinated. Appressoria were differentiated at this stage and, when infection of the first cell had already occurred, an infection vesicle and subjacent nascent hyphae were also observed (Fig. 1b). Haustoria were visible from 6-24 hai, indicative of biotrophic growth (Fig. 1c-e). At 18 hai, $P$. palmivora hyphae grew parallel to the cell files in the root cortex, forming a clear colonisation front between infected and non-infected tissues. In addition, extraradical hyphal growth was observed near the root tip (Fig. 1d). First sporangia occurred at 30 hai (Fig. 1f). Consistent with the symptoms observed on aerial parts, hypocotyl colonisation occurred between 30 hai and 48 hai (Fig. 1g). Finally, the presence of empty or germinating sporangia at 72 hai suggests possible secondary infections (Fig. 1h). Therefore, the P. palmivora asexual life cycle completes within 72 hai in $N$. benthamiana roots.

We supported our microscopic studies with biomass quantification based on transcript levels of the P. palmivora $40 \mathrm{~S}$ ribosomal protein S3A (WS21) (Fig. 1i). We further characterised the different stages observed microscopically by quantifying expression of the $P$. infestans orthologs of Hmp1 (haustorium-specific membrane protein) [51] (Fig. 1j) and the cell-cycle regulator $\mathrm{Cdc14}$ [52] (Fig. 1k). Hmp1 transcripts peaked between 3 hai and 6 hai and then decreased at later stages. By contrast, Cdc14 transcripts increased at late time points (48 hai and 72 hai). Taken together, these results further support the conclusion that $P$. palmivora exerts a hemibiotrophic lifestyle in $N$. benthamiana roots.

\section{De novo assembly of $P$. palmivora transcriptome from mixed samples}

We performed dual sequencing permitting de novo assembly of a $P$. palmivora transcriptome as well as an assessment of transcriptional changes in both host and pathogen over time. We extracted RNA from infected and uninfected $N$. benthamiana roots at six time points matching the key steps of the interaction identified by microscopy: 6 hai, 18 hai, 24 hai, 30 hai, 48 hai and 72 hai and an axenically grown $P$. palmivora sample containing mycelia and zoospores (MZ). Using Illumina HiSeq 2500 paired-end sequencing, we obtained a relatively uniform read depth of 50-60 M reads per sample (Additional file 1: Table S1). To cover all possible transcripts, we reconstructed the $P$. palmivora transcriptome de novo, combining ex planta and in planta root 


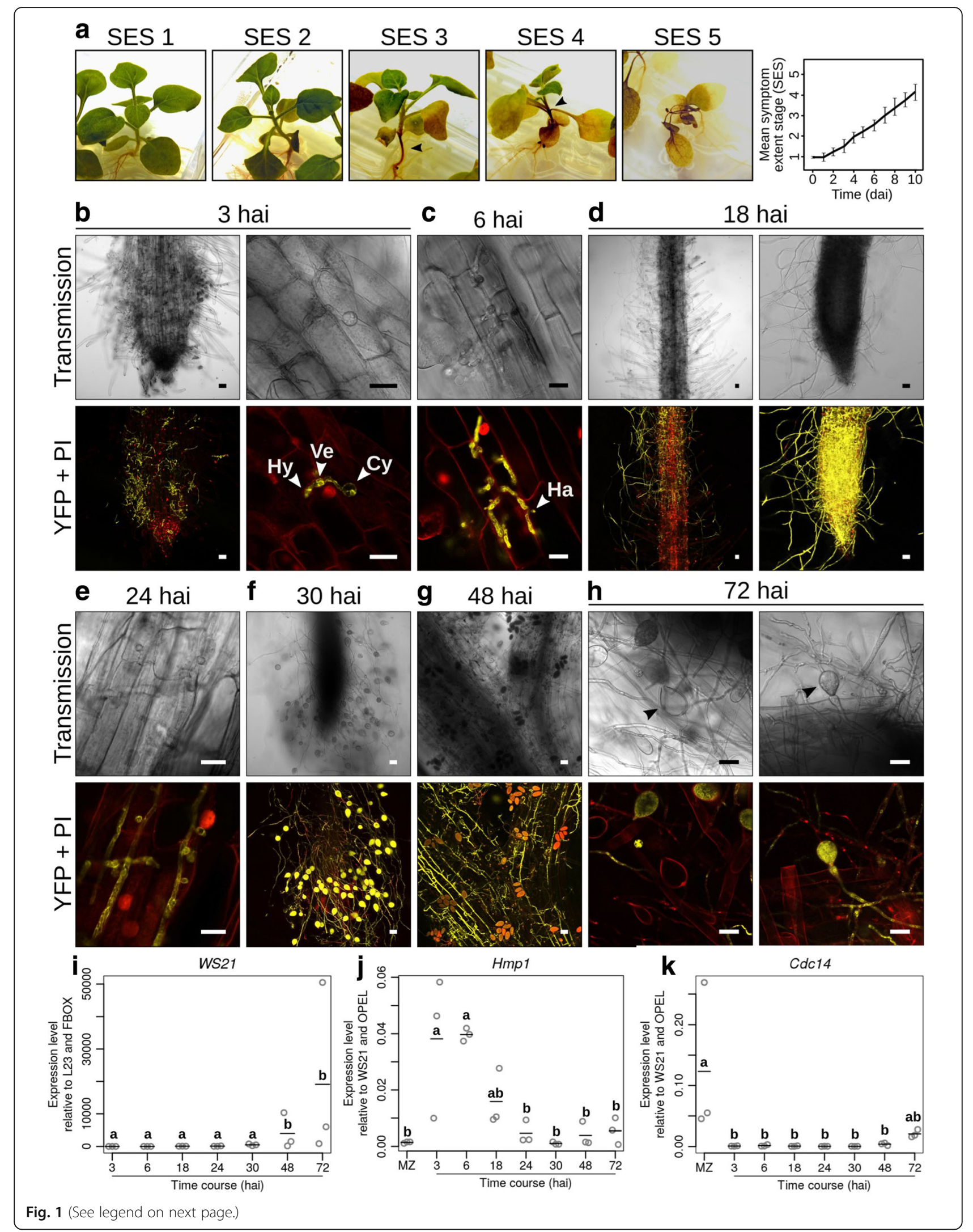


(See figure on previous page.)

Fig. 1 Phytophthora palmivora exerts a hemibiotrophic lifestyle in Nicotiana benthamiana roots. a Representative pictures of root-infected plantlets during P. palmivora infection, showing disease progression on the aboveground tissues. The successive symptom extent stages (SESs) were used to define a disease index in order to quantitate disease progression over time. $\mathbf{b}-\mathbf{h}$ Microscopic analysis of $\mathbf{N}$. benthamiana roots inoculated with transgenic $P$. palmivora LILI expressing an endoplasmic reticulum (ER)-targeted yellow fluorescent protein (YFP). Pictures were taken during penetration (b, 3 h after inoculation (hai)), early infection (c, 6 hai), biotrophy (d, 18 hai and $\mathbf{e}, 24$ hai), switch to necrotrophy (f, 30 hai) and necrotrophy (g, 48 hai and $\mathbf{h}, 72$ hai). Each panel shows transmission light (Transmission) and merged YFP fluorescence with propidium iodide (PI) staining (YFP + PI). Hy hypha, Ve vesicle, Cy cyst, Ha haustorium. Scale bar is $10 \mu \mathrm{m}$. i Quantification of $P$. palmivora biomass accumulation over time in N. benthamiana roots was measured by expression of $P$. palmivora WS21 relative to $N$. benthamiana $L 23$ and F-box reference genes. $\mathbf{j}, \mathbf{k}$ Expression of $P$. palmivora lifestyle marker genes $\mathrm{Hmpl}$ (j) and $\mathrm{Cdc} 14$ (k) were measured over time relative to P. palmivora WS21 and OPEL reference genes. Quantitative RT-PCR experiments were performed in triplicate. Circles represent values for each replicate. Bars represent the mean value. Statistical significance has been assessed using one-way analysis of variance (ANOVA) and Tukey's honestly significant difference (HSD) test $(p<0.05)$

samples as well as 76-nt Illumina paired-end reads from infected $N$. benthamiana leaf samples (more than $515 \mathrm{M}$ reads, Fig. 2a, Additional file 1: Table S1).

Following standard adapter trimming and read quality control, we applied a two-step filtering procedure (Fig. 2a) to separate pathogen reads from plant host reads. First we mapped the pooled read dataset to the $N$. benthamiana reference genome and collected unmapped read pairs. Recovered reads were subsequently mapped to the $N$. benthamiana transcriptome [53]. Reads not mapped to either host plant genome or transcriptome were used to run assemblies. Short reads (<60 nt) were filtered out to produce transcripts of better quality and coherence. Final de novo Trinity assemblies were run from $190 \mathrm{M}$ pre-processed, properly paired and cleaned reads (Additional file 1: Table S1). This yielded 57,579 'Trinity genes' corresponding to 100,303 transcripts with an average backwards alignment rate of $76 \%$, indicative of an overall acceptable representation of reads and therefore reasonably good assembly quality [54]. A total of 9491 Trinity genes (20,045 transcripts including all isoforms) were removed by additional checks for residual plant contamination, resulting in a final $P$. palmivora transcriptome of 48,089 Trinity genes

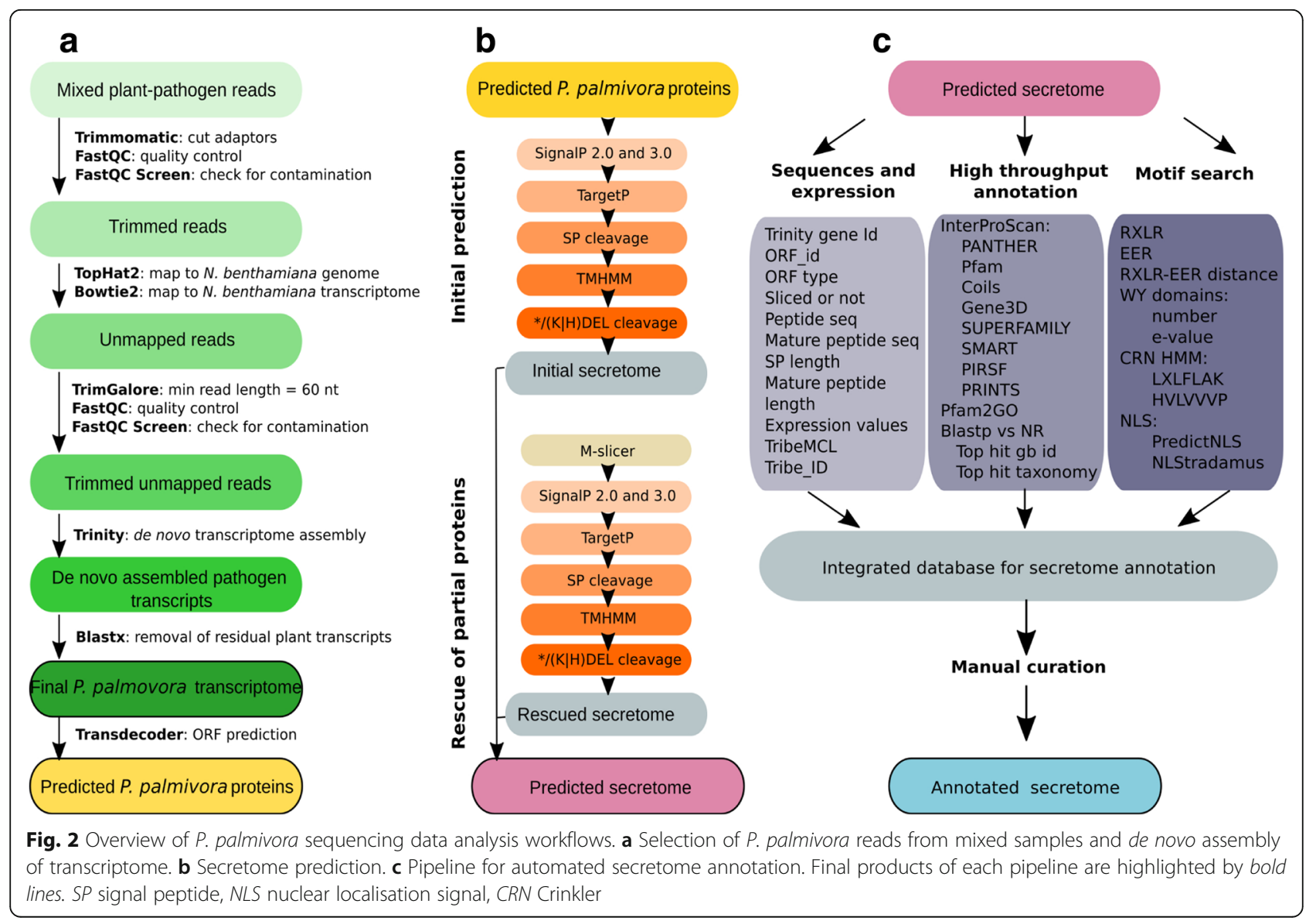


corresponding to 80,258 transcripts (Table 1). We further selected 13,997 Trinity genes (corresponding to 27,106 transcripts) having the best expression support (Additional file 2: Dataset 1).

We assessed completeness of P. palmivora assembly by benchmarking nearly universal single-copy orthologues (BUSCO) [55] (Table 1) and compared them to the BUSCO content of $P$. infestans, $P$. sojae and $P$. parasitica transcriptomes. We identified 326 BUSCO genes $(76 \%$ of eukaryotic BUSCO genes) in our P. palmivora assembly,

Table 1 De novo transcriptome assembly statistics for $P$. palmivora

\begin{tabular}{|c|c|}
\hline Metric & Value \\
\hline \multicolumn{2}{|l|}{ General assembly statistics } \\
\hline Total Trinity 'genes' & 48,088 \\
\hline Total Trinity transcripts & 80,258 \\
\hline Percent GC & 48.27 \\
\hline Smallest contig & 201 \\
\hline Largest contig & 217,111 \\
\hline \multicolumn{2}{|c|}{ Statistics based on all transcript contigs } \\
\hline Contig N10 & 6335 \\
\hline Contig N20 & 4510 \\
\hline Contig N30 & 3443 \\
\hline Contig N40 & 2621 \\
\hline Contig N50 & 1978 \\
\hline Median contig length & 282 \\
\hline Average contig length & 788 \\
\hline Total assembled bases & $63,247,196$ \\
\hline \multicolumn{2}{|c|}{ Statistics based on the longest isoform per gene } \\
\hline Contig N10 & 6738 \\
\hline Contig N20 & 4702 \\
\hline Contig N30 & 3604 \\
\hline Contig N40 & 2724 \\
\hline Contig N50 & 2003 \\
\hline Median contig length & 273 \\
\hline Average contig length & 765 \\
\hline Total assembled bases & $36,825,977$ \\
\hline \multicolumn{2}{|l|}{ Eukaryotic BUSCO genes } \\
\hline Complete single-copy BUSCOs & 256 \\
\hline Complete duplicated BUSCOs & 40 \\
\hline Fragmented BUSCOs & 30 \\
\hline Missing BUSCOs & 103 \\
\hline Total BUSCO groups searched & 429 \\
\hline Total BUSCO genes recovered & $326(76 \%)$ \\
\hline Phytophthora BUSCOs & 374 \\
\hline Updated \% recoverable BUSCOs & 87 \\
\hline
\end{tabular}

BUSCO benchmarking universal single-copy orthologues
348 (81\%) in P. infestans, $343(80 \%)$ in P. sojae and 360 (84\%) in P. parasitica (Table 1, Additional file 3: Figure S1). We also surveyed 14 publicly available Phytophthora genomes, yielding 20 additional BUSCO genes absent from all transcriptomes. Interestingly, the remaining 35 BUSCO genes were consistently missing from all analysed Phytophthora genomes and transcriptomes (Additional file 4: Table S2). These results suggest that our P. palmivora (LILI) transcriptome assembly actually contained $87 \%$ of BUSCO genes occurring in Phytophthora. Hence, our assembly shows acceptable quality and integrity and can be used as a reference for further studies.

\section{Clustering of plant and pathogen samples reflects different temporal dynamics during infection}

To explore the temporal expression dynamics of plant and pathogen genes, we separately mapped initial reads back to the reference $N$. benthamiana transcriptome (https:// solgenomics.net/) as well as to our P. palmivora transcriptome assembly (Additional files 5 and 6: Dataset 2 and Dataset 3). Principal component analysis (PCA) of plant samples revealed a major difference between infected and uninfected samples (91\% of variance; Fig. 3a). Plant transcript profiles from infected samples could be further assigned into three groups: 6 hai, $18-24-30$ hai and $48-$ 72 hai ( $4 \%$ of variance; Fig. 3a). Conversely, PCA analysis of $P$. palmivora transcript profiles identified two groups corresponding to early infection (6 to 24 hai) and late infection with MZ (48 and 72 hai), while 30 hai was kept apart (66\% of variance; Fig. 3b). Taken together, these results suggest different behaviour of plant and pathogen transcript profiles at the same times post-infection.

We identified 6590 plant and 2441 pathogen differentially expressed genes (DEGs) by performing differential expression analysis (log fold change (LFC) $\geq 2$, false discovery rate (FDR) $p<10^{-3}$ ) on all possible sample pairs (Fig. 3e, f, Additional file 3: Figure S4). Hierarchical clustering revealed 236 P. palmivora genes upregulated exclusively during biotrophy (from 6 to 30 hai), while all other stages shared sets of induced and expressed genes (Fig. 3f, d). Interestingly, major shifts in expression patterns occurred at 30 hai. Taken together with PCA grouping, this result suggests that 30 hai represents a transition stage from a biotrophic to a necrotrophic lifestyle.

In contrast to the pathogen, the plant transcriptome did not undergo sharp transitions over time and was instead characterised by steady up- or downregulation (Fig. 3e, c). Therefore, we utilised repeated upregulation of a gene in at least two time points as a selective criterion to alleviate the absence of replicates resulting in 2078 up- and 2054 downregulated genes. From these we validated 5 out of 6 genes with low or no expression under control conditions and high expression levels during infection using qRT-PCR 


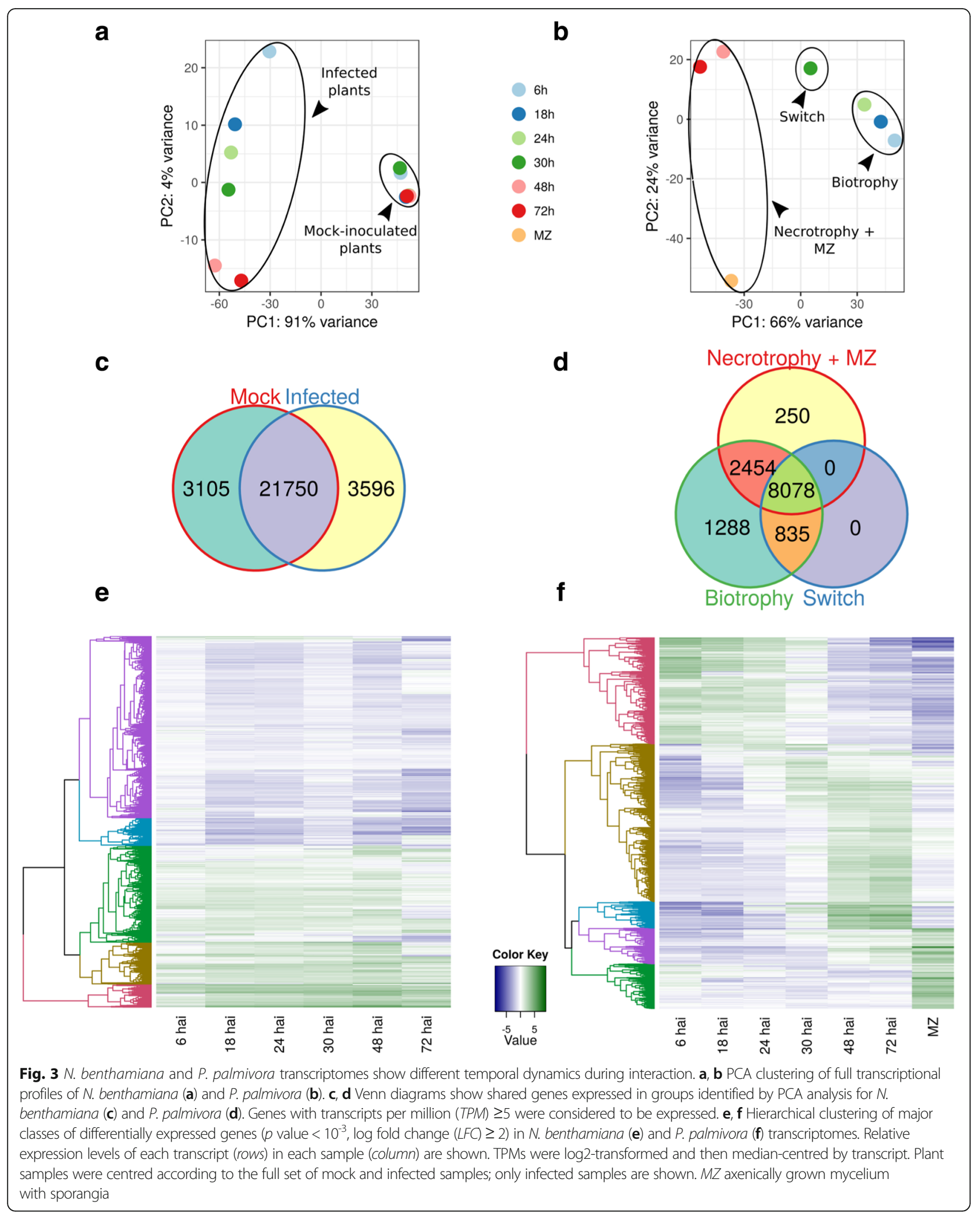


(Additional file 3: Figure S11). Gene ontology (GO) term analysis revealed that upregulated genes are enriched in biological processes related to hormone metabolism, abiotic stress (including oxidative stress, response to heat and wounding), defense, biosynthesis, transport, regulation of transcription and protein modification by phosphorylation and ubiquitination (Additional file 7: Dataset 4). Notably, we detected upregulation of numerous ethylene-responsive transcription factors (ERFs), indicating reprogramming of stress-specific defense regulation. Representatives of significantly enriched GO categories relevant for defense response include genes encoding endopeptidase inhibitors, such as Kunitz-type trypsin inhibitors. We also found upregulation of 48 genes encoding $O$-glycosyl hydrolases. In addition, we detected upregulation of trehalose biosynthesis pathway genes. Conversely, downregulated genes showed overall enrichment in biological processes associated with photosynthesis, cellulose biosynthesis and cell division. Taken together, these results suggest that infected $N$. benthamiana roots undergo major transcriptional and post-translational reprogramming leading to an overall activation of stress and defense responses.

\section{P. palmivora secretome prediction and annotation identify a set of effector candidate genes}

Pathogen-secreted effectors and hydrolytic enzymes are hallmarks of Phytophthora infection [56]. Therefore, we probed our $P$. palmivora transcriptome for transcripts encoding secreted proteins. A TransDecoder-based search for candidate open reading frames (ORFs) [57] identified 123,528 ORFs from predicted Trinity genes (isoforms included). We then analysed the predicted ORFs using an automated pipeline for secretome prediction (Fig. 2b) building on existing tools [58-60]. The pipeline was designed to predict signal peptides and cellular localisation with thresholds specific for oomycete sequences $[61,62]$ and to exclude proteins with internal transmembrane domains and/or an endoplasmic reticulum (ER) retention signal. We identified 4163 ORFs encoding putative secreted proteins.

Partial translated ORFs which were not predicted as secreted were subjected to an additional analysis (Mslicer) (Fig. 2b) and resubmitted to the secretome prediction pipeline. This improved procedure allowed us to rescue 611 additional ORFs encoding putative secreted proteins. In total, we identified 4774 ORFs encoding putative secreted $P$. palmivora proteins. We further selected a single representative secreted ORF for genes with sufficient expression support (transcripts per million (TPM) $\geq 1$ in three or more samples). This yielded 2028 P. palmivora genes encoding putative secreted proteins (Additional file 8: Dataset 5).
To maximise functional annotation of the $P$. palmivora secretome, we used an integrative approach (Fig. 2c) tailored to the use of known short motifs characteristic of oomycete secreted proteins. The pipeline contains three major blocks. The first block integrated all the sequence information, with assignment to 2028 nonredundant genes encoding secreted proteins as well as expression data. The second block combined results of homology searches, for both full-length alignments (blastn and blastx) and individual functional domains (InterProScan). The third block was designed to survey for known oomycete motifs and domains (such as RXLR, EER, WY for RXLR effectors; LXLFLAK for Crinklers and nuclear localisation signal (NLS) for effectors in general). The pipeline produced an initial secretome annotation (Fig. 2c) which was then manually curated to avoid conflicting annotations. This strategy allowed us to assign a functional category to 768 predicted secreted proteins (38\%) (Table 2).

Amongst predicted cytoplasmic effectors, the most prominent category encompasses 140 RXLR effectors. Of these 123 have a conserved RXLR motif followed by a dEER motif. WY domains were found in 30 RXLR-EER effectors and 3 RXLR effectors. Some RXLR effectors are unusually long ( $>400$ aa; the average length of RXLR effectors is 204 aa), suggesting that multiple effector domains are linked together. For instance, the effector domain of PLTG_07082 consisted of 8 internal repeats of a WY domain. It remains to be tested whether multiple WY domains within one effector fulfil different and independent roles.

Pfam searches revealed one full-length RXLR effector protein (PLTG_09049) carrying a C-terminal NUDIX domain. Pfam predictions assigned to partial genes identified two putative effectors, one bearing a NUDIX domain PF00293 (PLTG_05400) and the other a MYB/ SANT domain PF00249 (PLTG_06121).

Sequence similarity searches for RXLR effectors matching known oomycete avirulence proteins revealed PLTG_13552 as being similar to $P$. infestans AVR3a (PiAVR3a) (Additional file 3: Figure S2). Notably, P. palmivora AVR3a (PLTG_13552) harbours the K80/I103 configuration, but combined with a terminal valine instead of a tyrosine in PiAVR3a [63]. It thus remains to be tested whether PLTG_13552 is capable of triggering an R3a-mediated hypersensitive response.

Our pipeline only identified 3 genes encoding putative CRN effectors (PLTG_06681, PLTG_02753, PLTG_03744). Crinklers often lack predictable signal peptides but instead might be translocated into plant cells by an alternative mechanism [64]. An independent survey using hidden Markov model (HMM) prediction without prior signal peptide prediction revealed a total of 15 CRN motifcontaining proteins. Notably, the putative CRN effector 
Table 2 Representation of classes of putative extracellular proteins in P. palmivora secretome (strain LILI)

\begin{tabular}{|c|c|}
\hline Functional category & Number of proteins \\
\hline Effectors and effector candidates & 224 \\
\hline RXLR effectors & 140 \\
\hline RXLR-EER with WY domain & 30 \\
\hline RXLR-EER & 93 \\
\hline RXLR with WY domain & 3 \\
\hline RXLR only & 14 \\
\hline Crinklers & 15 \\
\hline Extracellular protease inhibitors (EPIS) & 28 \\
\hline Necrosis-inducing proteins (NLPs) & 24 \\
\hline Repeat-containing proteins & 17 \\
\hline Elicitins/elicitors & 42 \\
\hline $\begin{array}{l}\text { Lectins including cellulose binding elicitor } \\
\text { lectin (CBEL) }\end{array}$ & 25 \\
\hline$H x G P C E x \times x D D$ & 42 \\
\hline Small cysteine-rich proteins (SCRs) & 28 \\
\hline Cell wall-degrading enzymes (CWDEs) & 143 \\
\hline Glycosyl hydrolase & 80 \\
\hline Pectate lyase & 16 \\
\hline Pectin esterase & 8 \\
\hline Cellulase & 6 \\
\hline Glucosidase & 4 \\
\hline Polysaccharide lyase & 3 \\
\hline Other & 26 \\
\hline Cutinase & 3 \\
\hline Protease & 59 \\
\hline Serine protease & 27 \\
\hline Cysteine protease & 12 \\
\hline Metalloendopeptidase & 8 \\
\hline Aspartyl protease & 4 \\
\hline Other & 8 \\
\hline Oxidase & 45 \\
\hline Kinase & 41 \\
\hline Other enzymes & 158 \\
\hline Hypothetical & 1260 \\
\hline
\end{tabular}

PLTG_06681 carries a C-terminal serine/threonine kinase domain (PF00069) and shows low sequence similarity (34\%) to P. infestans effector CRN8 [65].

The $P$. palmivora secretome also contained a substantial number of apoplastic effectors (Table 2). We identified 28 genes encoding extracellular protease inhibitors, including extracellular serine protease inhibitors (EPIs) with up to five recognisable Kazal domains, several cystatins and cysteine protease inhibitors (EPICs) (Additional file 8: Dataset 5). PLTG_05646 encodes a cathepsin protease inhibitor domain followed by a cysteine protease and an ML domain (PF02221, MD-2-related lipid recognition domain). We also identified 28 proteins with small cysteine-rich (SCR) signatures, 18 of them being encoded in full-length ORFs, but only 6 in which the mature peptide is shorter than 100 aa. Longer SCRs can harbour tandem arrangements (PLTG_08623). In one case an SCR is linked to an N-terminal PAN/ APPLE domain, which is common for carbohydratebinding proteins [66].

Additionally the $P$. palmivora secretome contains 90 proteins carrying potential MAMPs, including necrosisinducing proteins (NLPs), elicitins and lectins. Out of 24 NLPs, 4 (PLTG_05347, PLTG_07271, PLTG_13864, PLTG_01764) carry a pattern of 20 amino acid residues which is similar to the immunogenic nlp20 motif (AiMYySwyFPKDSPVTGLGHR, less conserved amino acids in lower case) [67]. Transcripts encoding elicitins and elicitors in the $P$. palmivora secretome belong to the group of highest expressed ones during infection (Additional file 8: Dataset 5). We identified six transglutaminases, five of them (PLTG_04342, PLTG_02581, PLTG_10032, PLTG_10034 and PLTG_10033) carrying a conserved Pep-13 motif [28].

Despite the annotation efforts, $62 \%$ of the genes of the predicted secretome remained hypothetical and may contain novel classes of secreted proteins contributing to $P$. palmivora virulence. Inspection of the second largest tribe after RXLR effectors revealed 42 genes, all encoding a conserved $\mathrm{N}$-terminal domain with similarity to the PTHR13842 family combined with variable Cterminal domains. Motif enrichment analysis identified an invariant motif HxGPCExxxDD, encoded in the $\mathrm{N}$ terminal domain of all full-length transcripts (Additional file 3: Figure S3a and Additional file 8: Dataset 5). Homology modelling revealed structural similarity to lytic polysaccharide monooxygenases (Additional file 3: Figure S3b) [68]. However, a function for these proteins remains to be assigned. Given that their expression peaks during biotrophy (6-24 hai) and their conservation in 13 oomycete species (including representatives from genera Phytophthora, Plasmopara, Saprolegnia, Aphanomyces, Achlya, Thraustotheca and Albugo), they may represent a major class of apoplastic oomycete proteins.

Taken together, de novo transcriptome assembly followed by multistep prediction of ORF encoding potentially secreted proteins and a semi-automated annotation procedure allowed us to identify all major classes of effectors characteristic to oomycetes as well as $P$. palmivora-specific effectors with previously unreported domain arrangements. Our data suggest that $P$. palmivora's infection strategy relies on a diverse set of extracellular proteins, many of which do not match to previously characterised effectors. 
Most differentially expressed secreted proteins have their highest expression during biotrophy

In order to highlight dynamic expression changes of $P$. palmivora genes during infection, we performed fuzzy clustering of P. palmivora DEGs (Fig. 4) to lower sensitivity to noisy expression signals and to distinguish between expression profiles, even if they partially overlapped [69]. We identified 12 expression clusters falling into four main groups according to their temporal expression level maximum (Fig. 4a). Group A was composed of two clusters containing genes downregulated during infection. By contrast, expression levels of genes from group B peaked during biotrophy (6-24 hai). Group $C$ was composed of two clusters of genes for which transcripts accumulated mostly at 30 hai, while group D was formed of four clusters of genes with maximum expression during necrotrophy (48, 72 hai). Group B showed an overall enrichment in all classes of genes encoding secreted proteins (Fig. 4b), while groups A and C were enriched in elicitin-encoding genes. SCRs were enriched in group D. Also in group D and characterised by strong transcriptional induction was a gene (PLTG_02529) encoding several repetitions of an unknown Phytophthoraspecific amino acid motif. Expression dynamics of $18 P$. palmivora genes from different clusters were validated by qRT-PCR. Fourteen genes displayed expression patterns consistent with the results of in silico prediction (Additional file 3: Figure S5b-o). Taken together, these results suggest that $P$. palmivora transcriptome dynamics reflect the main lifestyle transitions observed by microscopic analysis of the infection process, and that a major upregulation of secreted proteins occurs during biotrophy in agreement with the occurrence of haustoria, which are a major site for pathogen secretion [13].

\section{Conserved RXLR effectors amongst $P$. palmivora isolates confer enhanced plant susceptibility to root infection}

We next focussed on the characterisation of four RXLR effectors upregulated during infection (Additional file 3: Figure S5) and named them REX1 (PLTG_01927 [GenBank:KX130348]), REX2 (PLTG_00715 [GenBank: KX130350]), REX3 (PLTG_00687 [GenBank:KX130351]) and REX4 (PLTG_13723 [GenBank:KX130352]). REX1-4 sequences from $P$. palmivora isolates with diverse geographic and host species origin (Additional file 9: Table S4) were obtained by PCR and amplicon sequencing. Primers specific for REX1-4 generated amplicons from at least 13 of the 18 isolates (REX1: 15, REX2: 15, REX3: 16, REX4: 13, Additional file 3: Figure S6) encoding proteins with high levels of amino acid sequence conservation. In particular, REX2 and REX3 were almost invariant, with one and two amino acid substitutions, respectively (Additional file 3: Figure S7).
$\mathrm{N}$-terminal translational green fluorescent protein (GFP) fusions of FLAG-tagged REX coding sequences (referred to as GFP:FLAG-REX1-4) expressed in roots of stable transgenic $N$. benthamiana plants (Fig. 5, Additional file 3: Figure S8) or transiently in the leaf epidermis (Additional file 3: Figure S9a-d) showed nuclear and cytoplasmic fluorescence at 24 hai originating from expression of full-length GFP:FLAG-REX1, 2 and REX4 protein fusions (Additional file 3: Figure S9e). In contrast to the other three, GFP:FLAG-REX3 fluorescence signals were much weaker in the leaf epidermis nucleus compared to the cytoplasmic signals and absent from root nuclei (Fig. 5c, Additional file 3: Figure S9c).

To determine the contribution of REX1-4 to $N$. benthamiana root infection, we then challenged hydroponically grown transgenic plants expressing GFP:FLAGREX1-4 or GFP16c-expressing plants (ER-targeted GFP) with P. palmivora zoospores (Fig. 6a, b) and monitored disease progression into aerial tissues over time using a disease index ranking from 1 to 5 derived from the symptoms previously reported (Fig. 1). Transgenic plants expressing GFP:FLAG fusions of the highly conserved REX2 and REX3 effectors displayed significantly accelerated disease symptom development ( $p$ alues of 5.4 10-16 and 0.013 , respectively) compared to GFP16c control plants, while expression of GFP:FLAG-REX1 and GFP:FLAGREX4 did not enhance susceptibility ( $p$ values of 0.66 and 0.24, respectively) (Fig. 6a, b).

\section{REX3 impairs plant secretion processes}

Suppression of defense component secretion has previously been found to be targeted by at least two mechanisms [48, 49]. We thus investigated the ability of the infectionpromoting REX2 and REX3 effectors to suppress host secretion (Fig. 6c). We generated pTrafficLights, a vector which enables expression of a secreted GFP (SPPR1-GFP) together with a nuclear-cytoplasmic DsRed from the same A. tumefaciens transfer DNA (Additional file 3: Figure S10a) and performed $A$. tumefaciens-mediated transient expression assays in $N$. benthamiana leaves using the same conditions as those of Bartetzko and co-workers [70]. Under control conditions, $\mathrm{SP}_{\mathrm{PR} 1}-\mathrm{GFP}$ is secreted to compartments with acidic $\mathrm{pH}$, preventing it from fluorescing, and we observed only a faint signal from the perinuclear endomembrane compartments (Additional file 3: Figure S10b). The GFP fluorescence signal intensity and distribution were altered by treatment with the secretion pathway inhibitor brefeldin A (BFA); this resulted in the formation of GFP-positive BFA bodies (Additional file 3: Figure $\mathrm{S} 10 \mathrm{~b})$. Co-expression of $\mathrm{SP}_{\mathrm{PR} 1}$-GFP with FLAGREX2 did not affect GFP levels, while FLAG-REX3 enhanced GFP levels in perinuclear endomembrane compartments and resulted in a strong labelling of the cortical ER (Fig. 6c). The ability of REX3 to retain GFP in 


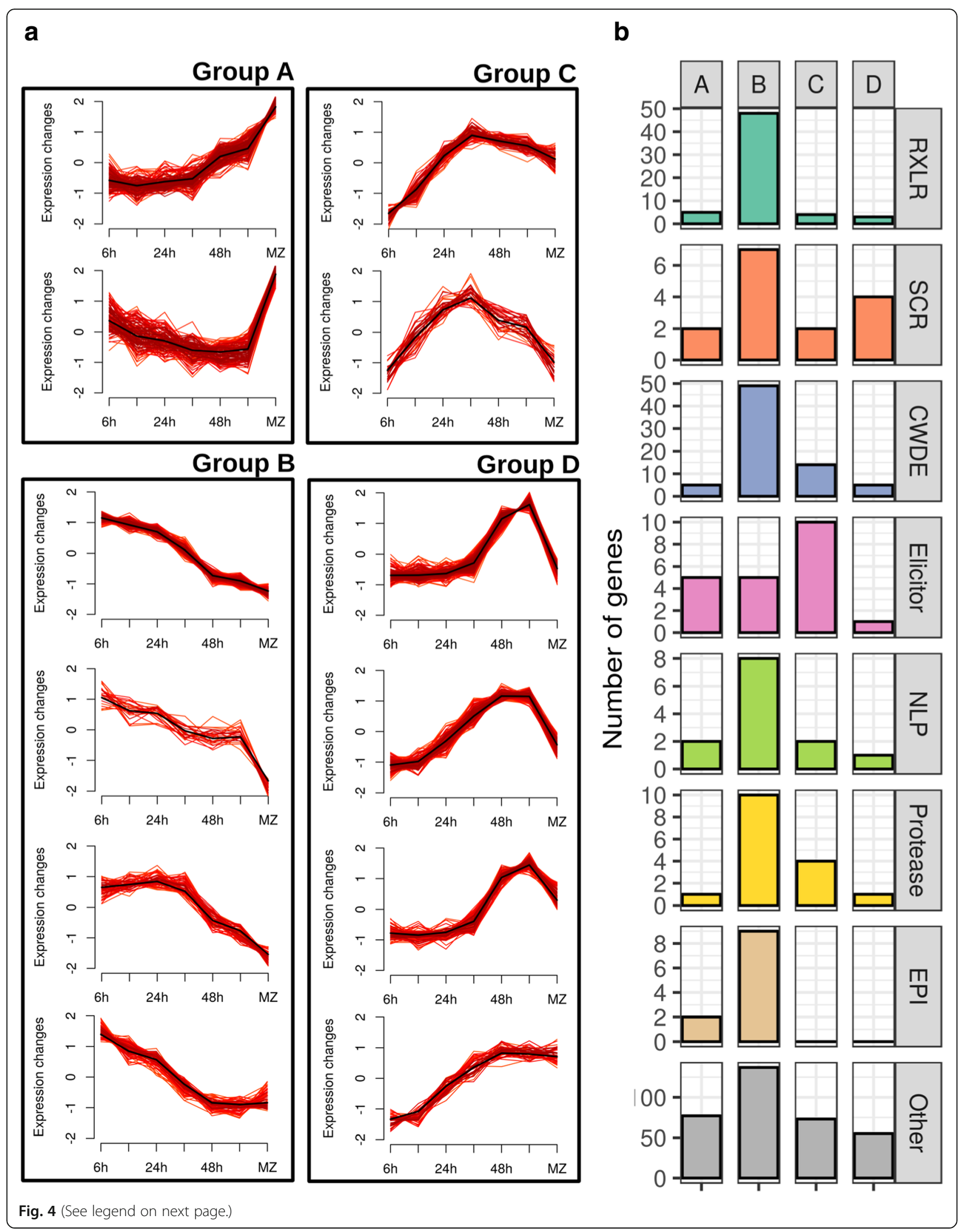


(See figure on previous page.)

Fig. 4 Temporal dynamics of $P$. palmivora differentially expressed genes (DEGS) during infection time course. Fuzzy clustering was performed on P. palmivora DEGs. Only genes with cluster membership values $\geq 0.7$ are shown, i.e. alpha cores (a). Functional distribution of secreted proteins for the grouped clusters is shown in (b). RXLR RXLR-effector, SCR small cysteine-rich protein, CWDE cell wall-degrading enzyme, NLP necrosis inducing protein, EPI protease inhibitor, Other other genes encoding proteins predicted to be secreted without specific functional category assigned

endomembrane compartments suggest that this effector may promote infection by interfering with host secretion pathways.

\section{The TIPTOP promoter is activated at root tip infection sites}

When screening our data for plant promoters responding early to $P$. palmivora attack. we found Niben101Scf03747g00005, encoding a small secreted protein containing two repeats of a conserved SGPSGxGH motif known from pathogen-associated molecular pattern (PAMP)-induced peptides (PIP/PIP-like; Additional file 3: Figure S12) [32] to be one of the most strongly induced plant genes. To study the spatial distribution of its promoter activity, we generated transgenic $N$. benthamiana plants expressing a promoter-GFP:uidA reporter fusion and challenged them with $P$. palmivora LILI-td [71] expressing a red fluorescent protein. Consistent with the transcriptomic data, histochemical betaglucuronidase (GUS) staining revealed a localised GUS signal at the tip of infected roots (Fig. 7) only where zoospores had accumulated but not in uninfected roots. We therefore termed the gene TIPTOP (Tip Induced Plant Transcript switched On by P. palmivora). TIPTOP promoter activation is correlated with $P$. palmivora infection (Fig. 7b). P. palmivora-triggered TIPTOP promoter activation was strongest adjacent to invasive hyphae as revealed by GFP confocal fluorescence microscopy (Fig. 7c). In addition, the TIPTOP promoter was not activated by abiotic stresses (cold, heat and $1 \mathrm{M}$ sodium chloride) and wounding, but weak activation was observed in root tips in response to flagellin (flg22) treatment (Additional file 3: Figure S13). PlantPAN 2 [72] analysis of the TIPTOP promoter sequence identified various transcription factor binding motifs (Additional file 10: Table S5). Taken together, these results suggest that TIPTOP is a root tip-specific $P$. palmivora-induced promoter.

\section{Discussion}

We utilised a dual transcriptomics approach coupled to a semi-automatic secretome annotation pipeline to study the interaction between $P$. palmivora and $N$. benthamiana roots. While the pathogen transcriptome undergoes remarkable shifts in expression patterns throughout the infection, we see a steady response of the plant transcriptome with no detectable major shifts in sets of differentially expressed genes. We used our dataset to identify $P$. palmivora and $N$. benthamiana genes implicated in the interaction, uncovered a new class of HxGPCE effector candidates and characterised two conserved biotrophic $P$. palmivora effector proteins which confer enhanced infection susceptibility when expressed in planta. We show that one of them, REX3, suppresses plant secretion processes. Surveying the set of early transcriptionally activated plant genes resulted in the identification of an $N$. benthamiana gene that is specifically induced at infected root tips and encodes a peptide with danger-associated molecular features.

\section{Dual transcriptomics and de novo assembly enable functional studies of unsequenced genomes}

Dual transcriptomics captures simultaneous changes in host and pathogen transcriptomes [73, 74] when physical separation of two interacting organisms is unfeasible. The diversity of plant pathogens often results in the absence of microbial reference genomes. This is particularly relevant for obligate biotrophic plant pathogens, which cannot be cultivated separately from their host. Our established viable alternative, a de novo assembly of a plant pathogen transcriptome from separated mixed reads followed by a semi-automated annotation, is thus applicable to a broader community. Taking advantage of the availability of the host reference genome, we separated $P$. palmivora reads from the mixed samples and combined them with reads from the ex planta samples to create a single de novo assembly for the pathogen transcriptome.

Assembly completeness in terms of gene content might be assessed based on evolutionary expectations, so that recovery of conserved genes serves as a proxy measure for the overall completeness (CEGMA [75] and BUSCO [55]). Our P. palmivora de novo assembly had sufficient read support (on average $76 \%$ reads mapping back), so we further probed it for the presence of BUSCOs. Since there is no specific oomycete set, we checked presence of 429 eukaryotic BUSCO genes and found 326 of them (76\%). Lack of some BUSCO genes in our assembly might result from the fact that originally BUSCO sets were developed to estimate completeness of genomic assemblies and did not require expression evidence [55]. To verify this, we extended the same completeness analysis to existing Phytophthora genomes and transcriptomes and found that transcriptomes in general indeed contained fewer BUSCOs. Moreover, we found 35 eukaryotic BUSCO genes consistently missing from Phytophthora genomic assemblies. Therefore, a BUSCO- 


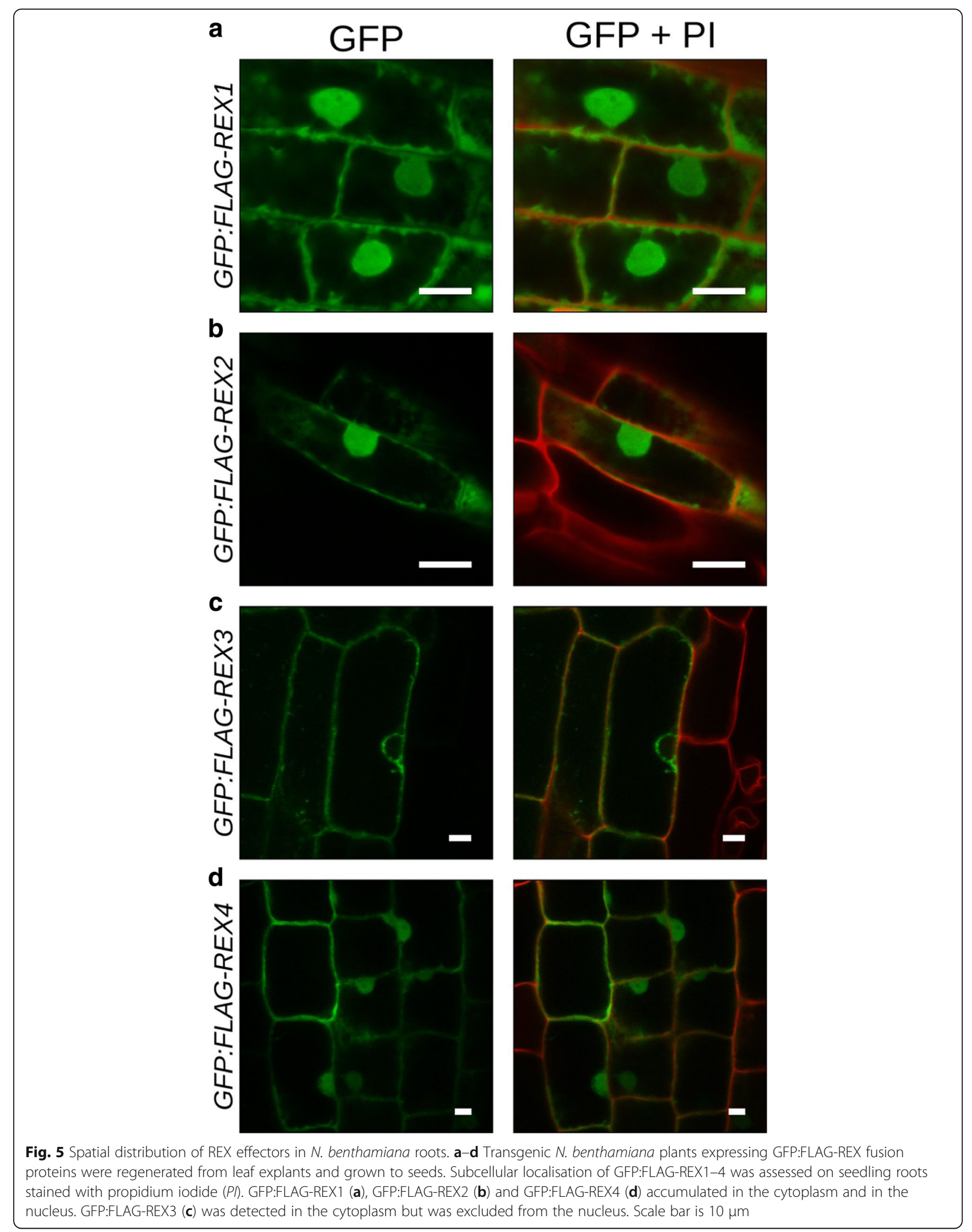



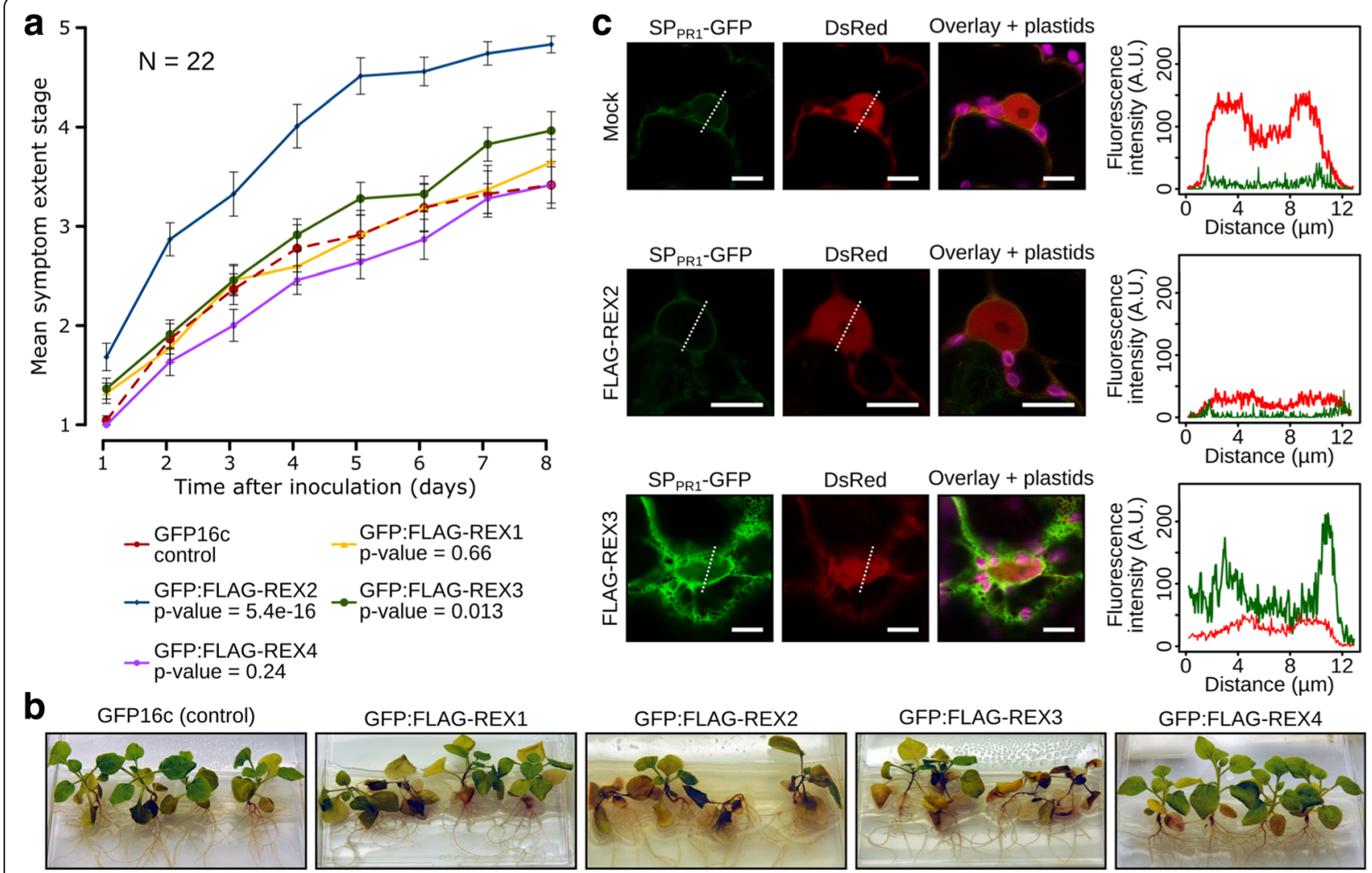

GFP:FLAG-REX4

Fig. 6 REX2 and REX3 increase N. benthamiana susceptibility to P. palmivora, and REX3 interferes with host secretion. Transgenic N. benthamiana plants expressing GFP16C (control) or GFP:FLAG-REX1 to GFP:FLAG-REX4 were challenged with zoospores from P. palmivora YKDEL, and disease progression was ranked over time using the previously defined symptom extent stages (SESS). a Representative disease progression curves for transgenic plants expressing GFP:FLAG-REX1 (yellow), GFP:FLAG-REX2 (blue), GFP:FLAG-REX3 (green) or GFP:FLAG-REX4 (magenta) compared to GFP16c control plants (red dashed). $p$ values were determined based on Scheirer-Ray-Hare nonparametric two-way analysis of variance (ANOVA) for ranked data. The experiment was carried out in duplicate $(N=22$ plants). b Representative pictures of infected plants, 8 days after infection. c Disease-promoting effectors REX2 and REX3 were co-expressed with a secreted GFP construct (SPPR1-GFP) in N. benthamiana leaves. GFP fluorescence was quantified along the nucleus

based completeness test for transcriptomes should be applied with caution within the Phytophthora genus, considering adjustments for expression support and the uneven distribution of eukaryotic single-copy orthologues. We propose that, with an ever-growing body of oomycete genomic and transcriptomic data, a specific set of benchmarking orthologues needs to be created to support de novo assemblies and facilitate studies of these economically relevant non-model plant pathogens [76].

So far, dual transcriptomics has only been used with limited time resolution and sequencing depth in plantpathogenic oomycete studies [77, 78]. Our study encompasses the full range of $P$. palmivora sequential lifestyle transitions occurring in $N$. benthamiana root, allowing reconstruction of a comprehensive transcriptional landscape in both interacting organisms. We found three major waves of $P$. palmivora gene expression peaks that correlate with its major lifestyle transitions: (1) early infection and biotrophic growth inside host tissues; (2) switch to necrotrophy; (3) late necrotrophy and sporulation. Similar transcription dynamics following switches of lifestyles were previously described for the hemibiotrophic pathogens Colletotrichum higginsianum [79], Phytophthora parasitica [80] during Arabidopsis root infection and $P$. sojae upon infection of soybean leaves [81], though the exact timing of infection was different.

Interestingly, the $N$. benthamiana transcriptional response to infection does not mirror the observed significant shifts in infection stage-specific $P$. palmivora gene expression. Instead it is characterised by steady induction and repression. High-resolution transcriptomics were applied to A. thaliana leaves challenged with $\mathrm{Bo}$ trytis cinerea to untangle the successive steps of host response to infection [82]. However, in the absence of pathogen expression data, it is not possible to correlate these changes with changes in the pathogen transcriptome. It is likely that pathogen expression patterns are not useable to infer a link to corresponding plant responses.

The response of $N$. benthamiana roots to P. palmivora is characterised by an upregulation of genes associated with hormone physiology, notably ethylene through 

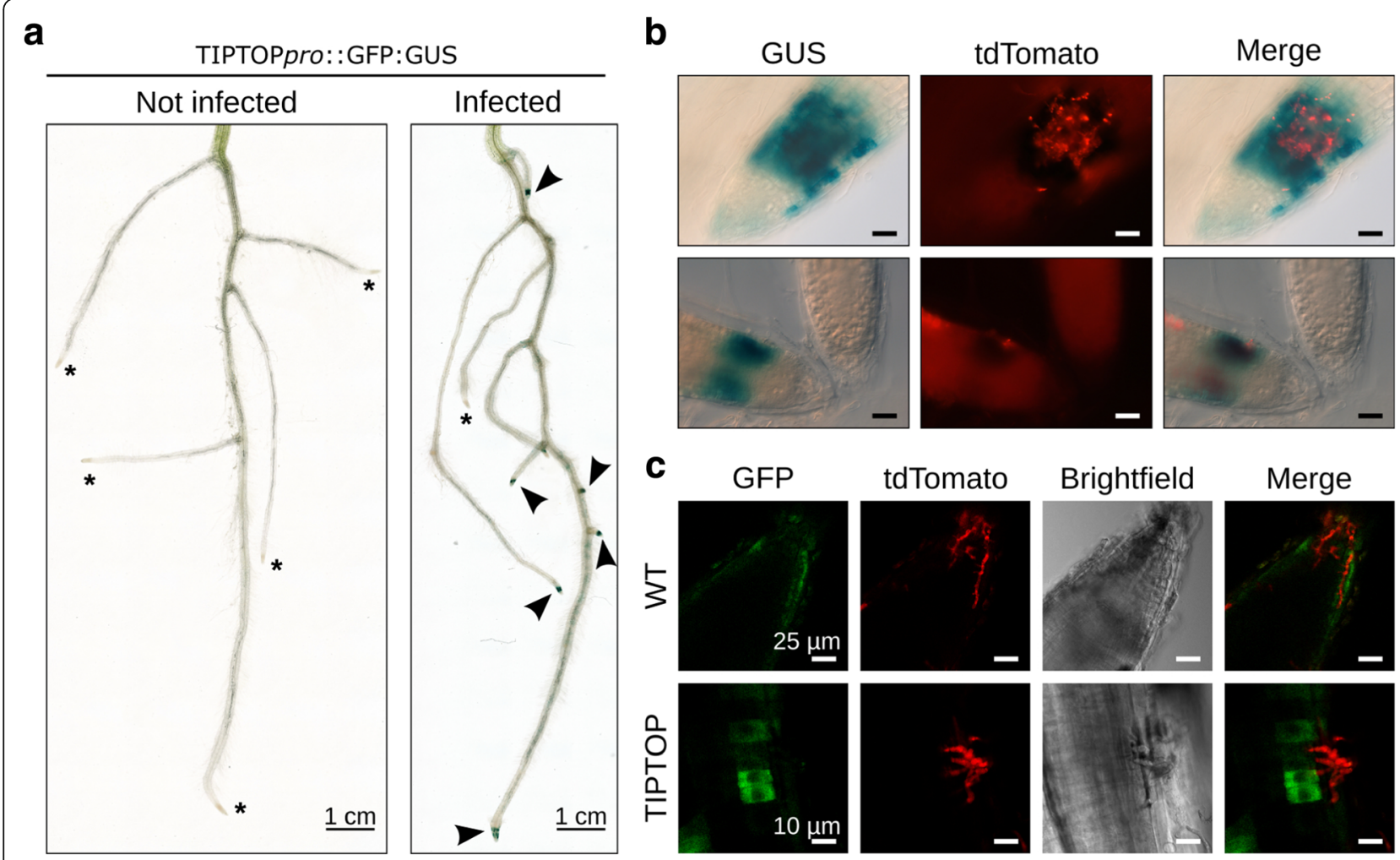

Fig. 7 The promoter of a gene encoding the secreted peptide TIPTOP is upregulated during early biotrophy in $\mathrm{N}$. benthamiana roots. a Representative pictures of beta-glucuronidase (GUS)-stained whole root systems of N. benthamiana transgenics carrying TIPTOPpro::GFP:GUS, non-infected or $16 \mathrm{~h}$ after infection with P. palmivora LILI-tdTomato. Stars represent unstained root tips. Arrowheads represent stained root tips. b Representative pictures of infected root tips after GUS staining, showing GUS signal at the vicinity of infection sites (top panels). Uninfected root tips from the same plant do not show any staining (bottom panels). Scale bar is $25 \mu \mathrm{m}$. c Representative pictures of GFP signal at the root tip of infected N. benthamiana transgenics expressing GFP:GUS fusion under the control of TIPTOP promoter

activation of ethylene response transcription factors (ERFs) and 1-aminocyclopropane-1-carboxylate (ACC) synthase. Ethylene is involved in $N$. benthamiana resistance to $P$. infestans [83]. We also observed an induction of two PIN-like auxin efflux carriers. Suppression of the auxin response was associated with increased $A$. thaliana susceptibility to $P$. cinnamomi disease and was stimulated by phosphitemediated resistance [84]. Interestingly, phosphite was also required for defense against $P$. palmivora [11]. We found upregulation of chitinases and endopeptidase inhibitors, such as Kunitz-type trypsin inhibitors, which are often induced by oomycete and fungal pathogens [85-87]. Induction of genes encoding $O$ glycosyl hydrolases is associated with cell wall remodeling, while phenylalanine ammonia lyases (PALs) contribute to cell wall reinforcement by activation of lignin biosynthesis $[88,89]$. Upregulation of the trehalose biosynthesis pathway is associated with membrane stabilisation [90] and partially mitigates the toxic effects of oxidative stress [91]. Upregulation of several enzymes of the mevalonate pathway suggests modulation of the biosynthesis of isoprenoids such as defense-associated phytoalexins as well as sterols. In particular, transcriptional repression of genes encoding sterol 4-alpha-methyl-oxidase 2-1 and C5 sterol desaturases suggests an attenuation of the brassinosteroid synthesis, while repression of genes with homology to sterol methyl transferase 2 points to a repression of the beta-sitosterol/stigmasterol branch. Conversely, induction of terpenoid synthases/ epi-aristolochene synthases points to a selective induction of the sesquiterpenes, which contain defenseassociated phytoalexins such as capsidiol [92, 93]. Finally, the $N$. benthamiana response to $P$. palmivora also includes upregulation of genes encoding late embryogenesis abundant (LEA) proteins as well as heat shock proteins. LEA proteins have been associated with the drought response [94, 95], and upregulation of genes associated with water deprivation upon Phytophthora infection has been previously reported [78]. Conversely, downregulated genes were mostly associated with photosynthesis, cellulose biosynthesis and cell division. These results were consistent with those of previous reports [96, 97]. 


\section{Analysing partial transcripts improved the predicted $P$. palmivora secretome}

To study $P$. palmivora secreted proteins, we developed a prediction and annotation pipeline tailored for signal peptide prediction based on ORFs derived from de novo assembled transcripts. Often a six-frame translation is utilised to identify candidate ORFs. However, we use a TransDecoder approach which enriches for the most likely translated ORF by integrating homology-based matches to known Pfam domains and Phytophthora proteins. Compared to sixframe translation, this approach can result in partial ORFs, which may lead to a misprediction of translation start sites and therefore of signal peptides. To overcome this, we implemented a refinement step in our secretome prediction pipeline to rescue partial ORFs by finding the next likely translation start position and following the secretome prediction steps. This procedure allowed us to rescue an additional 611 ORFs, including several which likely encode RXLR effectors, elicitins and cell wall-degrading enzymes, thus highlighting the importance of this additional step.

\section{Effector-guided resistance breeding potential}

We identified two RXLR effectors that show high sequence conservation amongst $P$. palmivora isolates worldwide, which suggests that they may represent core effectors that cannot be lost or mutated without a fitness cost for the pathogen [21]. As such, these effectors constitute valuable candidates to accelerate cloning of disease resistance $(R)$ genes and effector-assisted deployment of resistance. This strategy has been used against $P$. infestans [22].

Our approach identified a potential AVR3a homologue in $P$. palmivora (PLTG_13552). The P. infestans AVR $3 \mathrm{a}^{\mathrm{KI}}$ allele confers avirulence to $P$. infestans isolates on $R 3 a$-expressing potatoes, while the AVR $3 \mathrm{a}^{\mathrm{EM}}$ allele is not being recognised [63]. It will be interesting to study whether potato $R 3 a$ or engineered $R 3 a$ derivatives with a broader recognition spectrum $[98,99]$ can be exploited to generate resistance towards $P$. palmivora in economically relevant transformable host plants. Additionally, $P$. palmivora proteins also harbour Pep-13-type MAMP motifs present in four transglutaminases and several nlp20-containing NLPs. While the Pep-13 plant receptor remains to be found, the receptor-like kinase RLP23 has recently been identified as an nlp20 receptor [100] with the potential to confer resistance even when transferred into other plant species. Introduction of RLP23 into P. palmivora host plants may thus be another strategy to engineer resistant crops.

\section{The $P$. palmivora effector REX3 inhibits plant secretion pathways}

We found that REX3 interferes with host secretion, a common strategy of bacterial and oomycete pathogens
$[49,70]$. Rerouting of the host late endocytic trafficking to the extrahaustorial membrane $[41,101]$ and accumulation of the small GTPase RAB5 around haustoria [42] is well documented. Given that REX3 is almost invariant in $P$. palmivora, it is likely that REX3 targets components of the secretory pathway which are conserved among diverse host species. Of the four functionally tested RXLR effectors, the two most conserved ones (REX2, REX3) amongst P. palmivora isolates both conferred increased susceptibility. REX2 and REX3 therefore represent important targets for disease resistance breeding in tropical crops. It is possible that isolate-specific variants of REX1 and REX4 may provide a colonisation benefit only in hosts other than $N$. benthamiana.

\section{P. palmivora triggers expression of danger-associated molecular pattern peptides}

Upon $P$. palmivora root infection, $2886 N$. benthamiana genes were upregulated and 3704 genes downregulated. Compared to previously studied root transcriptomes of responses to broad-host-range Phytophthora species [96, 102], our data permitted the identification of early induced genes such as TIPTOP, a P. palmivora-responsive root tip promoter. An exciting future perspective is its exploitation for induced early resistance against Phytophthora root infections. This promoter also provides inroads to dissect early host cell responses to $P$. palmivora, when employed in combination with a cell sorting approach to generate samples enriched for infected cells.

The TIPTOP gene encodes a peptide with similarities to DAMP peptides [103]. The occurrence of two tandem repeats of a conserved sGPSPGxGH motif in the TIPTOP protein is reminiscent of the SGPS/GxGH motifs of PIP and PIPL peptides [32, 104], and the closest Arabidopsis homologues of TIPTOP, PIP2 and PIP3, are implied in responses to biotic stress.

Hou and co-workers showed that the PIP1 peptide is induced by pathogen elicitors and amplifies $A$. thaliana immune response by binding to the receptor-like kinase 7 (RLK7) [32]. Analysis of in silico data showed that PIP2 and PIP3 were activated upon A. thaliana infection by Botrytis cinerea or P. infestans [104]. In contrast to PIP1 and PIP2, the TIPTOP promoter is inactive under control conditions, suggesting it may undergo a different transcriptional regulation than the previously characterised Arabidopsis peptides.

\section{Conclusions}

The use of dual transcriptomics represents a successful approach to identify transcriptionally regulated effectors as well as plant genes implicated in the root infection process. We found conserved MAMPs and effectors with similarity to known AVR proteins such as AVR3a which may harbour the potential for disease resistance 
engineering. We characterised two conserved RXLR effectors conferring enhanced susceptibility to root infection and confirmed interference with host secretion as a $P$. palmivora pathogenicity mechanism. Furthermore, the $P$. palmivora-inducible TIPTOP promoter and the PIP2, 3-like peptide are promising leads for engineering $P$. palmivora resistance. In summary, our findings provide a rich resource for researchers studying oomycete plant interactions.

\section{Methods}

\section{Plant material and growth conditions}

$N$. benthamiana seeds were surface sterilised for $3 \mathrm{~min}$ with $70 \%$ ethanol and $0.05 \%$ sodium dodecyl sulfate (SDS) and rinsed twice in sterile water. Seeds were coldstratified for 2 days and sown on Murashige and Skoog (MS) medium (Sigma-Aldrich Chemical Company, St Louis, MO, USA) supplemented with $20 \mathrm{~g} / \mathrm{L}$ sucrose and $10 \mathrm{~g} / \mathrm{L}$ agar. For in vitro susceptibility assays, 2-week-old plants were transferred to square Petri dishes using the hydroponics system described elsewhere [105]. These dishes, each containing five plants, were then placed slanted for 2 weeks at $25{ }^{\circ} \mathrm{C}$ under a 16-h photoperiod. For inoculations, zoospore suspension was added directly to the root compartment containing the liquid medium.

\section{$P$. palmivora growth conditions and $N$. benthamiana root inoculation}

P. palmivora Butler isolate LILI (reference P16830) was initially isolated from oil palm in Colombia [71] and maintained in the $P$. palmivora collection at the Sainsbury Laboratory (Cambridge, UK). Transgenic P. palmivora LILI strains expressing KDEL-YFP [9] and tdTomato [71] have been previously described. Phytophthora growth conditions and the production of zoospores have been described elsewhere [10].

\section{Root inoculation and disease progression assays}

For the investigation of effector dynamics during infection and activation of the TIPTOP promoter, we added $10^{5} \mathrm{P}$. palmivora zoospores to the liquid medium of Petri dishes containing 20-day-old plantlets grown as described already. Root infection assays were adapted from the $A$. thaliana-P. parasitica infection system described by Attard and co-workers [105]. One-week-old N. benthamiana seedlings were grown on hard (2\%) agar strips with roots immersed in 1/10th liquid MS medium for 2 weeks. Plates were then inoculated with 500 zoospores of $P$. palmivora LILI KDEL-YFP. Plants were scored on a daily basis using a disease index composed of five symptom extent stages (SESs): healthy plants with no noticeable symptoms were given a SES value of 1 . Plants with at least one wilted leaf were given a SES value of 2. Plants showing a brownish, shrunken hypocotyl were given a SES value of 3. Plants showing a brownish, shrunken hypocotyl and stem with multiple invaded or wilted leaves were given a SES value of 4. Finally, dead plants were given a SES value of 5. Statistical analyses of disease severity were based on Scheirer-Ray-Hare nonparametric two-way analysis of variance (ANOVA) for ranked data ( $H$ test) [106].

\section{Quantitative reverse transcription-polymerase chain reaction (qRT-PCR) analyses}

Total RNA was extracted from frozen, axenically grown mycelium with sporangia (sample MZ) and infected roots harvested at $3,6,18,24,30,48$ and $72 \mathrm{~h}$ after inoculation (hai) using the RNeasy Plant Mini Kit (Qiagen, Germantown, MD, USA). One microgram was reverse transcribed to generate first-strand complementary DNA (cDNA), using the Roche Transcriptor First Strand cDNA Synthesis Kit according to the manufacturer's instructions (Roche, Basel, Switzerland). Quality was assessed by electrophoresis on agarose gel. qRT-PCR experiments were performed with $2.5 \mu \mathrm{l}$ of a 1:20 dilution of first-strand cDNA and LightCycler 480 SYBR Green I Master mix, according to the manufacturer's instructions (Roche). Gene-specific oligonucleotides were designed with BatchPrimer3 software [107] (Additional file 11: Table S3), and their specificity was validated by analysing dissociation curves after each run. Genes encoding the $P$. palmivora orthologues of $P$. parasitica elicitor OPEL and a 40S ribosomal subunit S3A (WS21) were selected as constitutive internal controls for $P$. palmivora genes [108]. Genes encoding L23 (Niben101Scf01444g02009) and FBOX (Niben101Scf04495g02005) were selected as constitutive internal controls for $N$. benthamiana genes [109]. Three biological replicates of the entire experiment were performed. Gene expression was normalised with respect to constitutively expressed internal controls, quantified and plotted using R software.

\section{Plasmid construction}

The vector pTrafficLights was derived from pK7WGF2 (Plant System Biology, Gent University, Belgium). A cassette containing the signal peptide sequence of Nicotiana tabacum pathogenesis-related protein 1 (PR-1 [GenBank:X06930.1]) fused in frame with the green fluorescent protein (GFP) was obtained by PCR using primers SP-F/SP-R (Additional file 11: Table S3) and ligated into pK7WGF2 using SpeI and EcoRI restriction enzymes. The AtUBQ10pro::DsRed cassette was amplified from pK7WGIGW2(II)-RedRoot (Wageningen University, Netherlands) using primers RedRoot-F/RedRoot-R (Additional file 11: Table S3) and ligated into pK7WGF2 using XbaI and BamHI restriction enzymes.

The TIPTOP promoter (1230 bp, ending 46 bp before start codon) was PCR-amplified from $N$. benthamiana 
genomic DNA using primers TIPTOP-F2/TIPTOP-R2 (Additional file 11: Table S3) and cloned into a pENTR/DTopo vector (Life Technologies Inc., Gaithersburg, MD, USA). The entry vector was then used for LR recombination (Life Technologies Inc.) into expression vector pBGWFS7 (Plant System Biology, Gent University, Belgium).

\section{Transient Agrobacterium tumefaciens-mediated expression} For transient expression of effectors in $N$. benthamiana leaves, A. tumefaciens cells (strain GV3101-pMP90) were grown overnight with appropriate antibiotics. The overnight culture was then resuspended in agroinfiltration medium composed of $10 \mathrm{mM} \mathrm{MgCl} 2,10 \mathrm{mM}$ 2-(N-morpholino)ethanesulfonic acid (MES) pH 5.7 and $200 \mu \mathrm{M}$ acetosyringone. The optical density at $600 \mathrm{~nm}\left(\mathrm{OD}_{600}\right)$ was then adjusted to 0.4 for transient expression of effectors. For secretion inhibition assays, effectors and pTrafficLights construct were mixed together in a 1:1 ratio to a final $\mathrm{OD}_{600}$ of 0.8 . Agroinfiltrations were performed after 3 -h-long incubation at $28{ }^{\circ} \mathrm{C}$ using a syringe without a needle on the abaxial side of 5 -weekold $N$. benthamiana leaves.

\section{Generation of transgenic Nicotiana benthamiana}

$N$. benthamiana stable transformation was performed according to [110] with the following modifications: leaf discs were incubated in shoot-inducing medium (SIM) composed of 1X Murashige and Skoog (MS) medium supplemented with $2 \%$ sucrose, $0.7 \%$ agar, $50 \mathrm{mg} / \mathrm{L}$ kanamycin, $50 \mathrm{mg} / \mathrm{L}$ carbenicillin, $500 \mathrm{mg} / \mathrm{L}$ timentin and a 40:1 ratio of 6-benzylaminopurine (BAP) and 1naphthaleneacetic acid (NAA). Emerging shoots were cut and transferred to root-inducing medium (RIM), which has the same composition as SIM without BAP. After the first roots emerged, plantlets were transferred to soil and grown at $25{ }^{\circ} \mathrm{C}$ under a 16 -h photoperiod.

\section{Histochemical staining for GUS activity}

Transgenic $N$. benthamiana plantlets carrying the pTIPTOPpro::GFP:GUS sequence were harvested $14 \mathrm{~h}$ after inoculation and incubated in a staining solution containing $100 \mathrm{mM}$ sodium phosphate $\mathrm{pH} 7.0,0.1 \%$ (v/v) Triton $\mathrm{X}-100,5 \mathrm{mM} \mathrm{K}_{3} \mathrm{Fe}(\mathrm{CN})_{6}, 5 \mathrm{mM} \mathrm{K} \mathrm{K}_{4} \mathrm{Fe}(\mathrm{CN})_{6}$ and $2 \mathrm{mM}$ 5-bromo-4-chloro-3-indoxyl- $\beta$-D-glucuronid acid (Xgluc). Staining was carried out for $3 \mathrm{~h}$ at $37{ }^{\circ} \mathrm{C}$. The plantlets were then washed with distilled water and observed with an AxioImager M1 epifluorescence microscope (Zeiss, Oberkochen, Germany) equipped for Nomarski differential interference contrast (DIC).

\section{Confocal microscopy}

Confocal laser scanning microscopy images were obtained with a Leica SP8 laser-scanning confocal microscope equipped with a $63 \times 1.2$ numerical aperture (NA) objective (Leica, Wetzlar, Germany). A white-light laser was used for excitation at $488 \mathrm{~nm}$ for GFP visualisation, at $514 \mathrm{~nm}$ for yellow fluorescent protein (YFP) visualisation and at $543 \mathrm{~nm}$ for the visualisation of tdTomato. Pictures were analysed with ImageJ software (http://imagej.nih.gov/ij/) and plugin Bio-Formats.

\section{Library preparation and sequencing}

$N$. benthamiana and $P$. palmivora messenger RNAs (mRNAs) were purified using Poly(A) selection from the total RNA sample and then fragmented. cDNA library preparation was performed with the TruSeq ${ }^{\circ}$ RNA Sample Preparation Kit (Illumina, San Diego, CA, USA) according to the manufacturer's protocol. cDNA sequencing of the 13 samples (MZ, infected $N$. benthamiana root samples and uninfected $N$. benthamiana plants) was performed in four lanes of Illumina NextSeq 2500 in a 100 paired-end mode. Samples were de-multiplexed and analysed further.

mRNAs from additional samples of a short leaf time course ( $P$. palmivora mycelium, $N$. benthamiana leaves 2 days after inoculation (dai) and $N$. benthamiana leaves 3 dai) were purified using $\operatorname{Poly}(\mathrm{A})$ selection from the total RNA sample. cDNA libraries were prepared using the NEBNext $^{\circ}$ RNA library preparation kit (New England Biolabs, Hitchin, UK) according to the manufacturer's protocol and sequenced on an Illumina GAII Genome Analyzer in a 76 paired-end mode in three separate lanes. Reads obtained from these three samples were used for $P$. palmivora de novo transcriptome assembly only. The raw fastq data are accessible at http://www.ncbi.nlm.nih.gov/sra/ with accession number SRP096022.

\section{De novo transcriptome assembly}

In order to capture the full complexity of the P. palmivora transcriptome, we pooled all the samples potentially containing reads from $P$. palmivora (Fig. 2): eight mixed (plant-pathogen, combining leaf and root infections), one exclusively mycelium and one mixed myceliumzoospores sample. Initial read quality assessment was done with FastQC (Babraham Bioinformatics, Cambridge, UK). Adapters were removed using Cutadapt [111]. To exclude plant reads from the library, raw paired reads were first aligned to the $N$. benthamiana reference genome (v1.01) using TopHat2 [112]. Unmapped reads (with both mates unmapped) were collected with SAMtools (samtools view -b -f 12 -F 256), converted to fastq with bedtools and processed further. To estimate the level of residual contamination by plant and potentially bacterial reads, the resulting set of reads was subjected to FastQ Screen against the UniVec database, all bacterial and archaeal sequences obtained from the RefSeq database, all viral sequences obtained from 
the RefSeq database, the $N$. benthamiana genome (v1.01), and subset 16 oomycete species (mostly Phytophthora species). Since the preceding test revealed substantial residual contamination by $N$. benthamiana reads, an additional round of bowtie 2 alignment directly to the $N$. benthamiana transcriptome [53] was performed followed by FastQ Screen. Reads not aligned to the $N$. benthamiana genome and transcriptome were further subjected to quality control using Trimmomatic (minimum read length $=60$ ). The quality parameters for the library were assessed using FastQC. All ( 190 M) filtered reads were subjected to de novo assembly with Trinity (Trinity v2.1.1) on a high-RAM server with minimal $\mathrm{k}$-mer coverage $=2$ and $\mathrm{k}$-mer length $=25$. In silico read normalisation was used due to the large number of input reads, in order to improve assembly efficiency and to reduce run times [57]. The resulting assembly was additionally checked for plant contamination using blastn search against the plant division of the National Center for Biotechnology Information (NCBI) RefSeq genomic database. Trinity genes having significant sequence similarity (e-value threshold $\leq 10^{-5}$ ) to plant sequences were removed from the resulting transcriptome. The final version of assembly included Trinity genes with sufficient read support.

\section{De novo assembly statistics and integrity assessment}

General statistics of the assembly were determined using the 'TrinityStats.pl' script provided with the Trinity release and independently using Transrate (http://hibberdlab.com/ transrate/) and Detonate (http://deweylab.biostat.wisc.edu/ detonate/) tools. Assembly completeness was estimated using the eukaryotic set of BUSCO profiles (v1) [55]. BUSCO analysis was performed for the full transcriptome assembly and for the reduced assembly, obtained after retaining only the longest isoform per Trinity gene. BUSCO genes missing from the assembly were annotated with InterProsScan based on the amino acid sequences emitted from the corresponding hmm profile ('hmmemit' function from hmmer package, http://hmmer.org/). Overall expression support per assembled transcript was performed after transcript abundance estimation. Trinity genes with TPM $\geq$ 1 in at least three samples were considered further.

\section{Protein prediction and annotation}

ORFs were predicted using TransDecoder software [57]. At the first step, ORFs longer than 100 aa were extracted. The top 500 longest ORFs were used for training a Markov model for coding sequences; candidate coding regions were identified based on log-likelihood score. Additionally all the ORFs having homology to protein domains from the Pfam database and/or $P$. sojae, $P$. parasitica, $P$. infestans and $P$. ramorum protein sequences downloaded from the Uniprot database (accession numbers: UP000005238, UP000006643,
UP000002640, UP000018817) were also retained (blastp parameters: max_target_seqs 1 -evalue 1e-5).

\section{Secretome prediction}

For the automatic secretome prediction a custom script was written, employing steps taken for $P$. infestans secretome identification [16]. Predicted proteins were subsequently submitted to SignalP 2.0 (Prediction = 'Signal peptide'), SignalP 3.0 (Prediction = 'Signal peptide', $Y \max$ score $\geq 0.5, D$ score $\geq 0.5, S$ probability $\geq 0.9$ ), TargetP (Location = 'Secreted') [113] and TMHMM (ORFs with transmembrane domains after predicted signal peptide cleavage site were removed) [114]. Finally, all proteins with terminal 'KDEL' or 'HDEL' motifs were also removed, as these motifs are known to be ER-retention signals [115]. Exact duplicated sequences and substrings of longer ORFs were removed to construct a non-redundant set of putative secreted proteins. Taking into account possible fragmentation of de novo assembled transcripts, a custom Python script (M-slicer) was developed to rescue partial proteins with mispredicted coding sequence (CDS) coordinates. The script takes as an input all the partial translated ORFs which were not predicted to be secreted initially and creates a sliced sequence by finding the position of the next methionine. The M-sliced proteins were subjected to the same filtering step as was done with the initial secretome. The same script, omitting the M-slicer refinement, was used to systematically predict $N$. benthamiana genes encoding putative secreted proteins.

\section{Secretome annotation}

To annotate putative secreted proteins a complex approach was used, combining several lines of evidence: (1) blastp search against the GenBank non-redundant (NR) database with e-value $\leq 10^{-6}$; (2) InterProScan (v5.16) search against databases of functional domains (PANTHER, Pfam, Coils, Gene3D, SUPERFAMILY, SMART, PIRSF, PRINTS) with default parameters [116]; (3) RXLR and EER motif prediction using regular expressions; (4) WY motif prediction based on WY-fold HMM by hmmsearch function from the HMM3 package (http://hmmer.org/); (5) LXLFLAK and HVLVVVP motif predictions based on HMM model build on sequences of known CRN effectors; (6) NLS motif prediction by NLStradamus [117] (v1.8, posterior threshold =0.6) and PredictNLS [118] with default parameters. The TribeMCL algorithm was used to cluster predicted putative secreted proteins with signal peptide and after signal peptide cleavage (mature proteins). The tribing results were used as a soft guidance for functional annotation (proteins belonging to the same tribe are likely to have the same function). All obtained data were aggregated in the Additional file 8: Dataset 5: Annotated P. palmivora secretome. Functional categories were assigned based on 
manual curation of the resulting table. A 'hypothetical' category was assigned to proteins either having similarity to only hypothetical proteins or when the top 20 hits of blastp output did not show consistency in terms of distinct functional categories. Proteins having significant sequence similarity to ribosomal, transmembrane proteins or proteins with known intracellular localisation (e.g. heat shock proteins) and/or having respective domains identified by InterProScan were marked as false predictions. A contamination category was assigned for proteins with significant sequence similarity (revealed by blastp) to amino acid sequences from phylogenetically distant taxa (e.g. plants or bacteria). Entries marked as both 'false prediction' or 'contamination' were excluded from the final secretome. Homology modelling was performed using SWISS-MODEL [119].

\section{Transcriptome annotation}

All the remaining predicted proteins were annotated by scanning against InterProScan databases of functional domains (v5.16-55) and by performing a blastp search against the GenBank NR database (download date: 06.01.2016) and published reference Phytophthora genomes. For transcripts without predicted ORFs a blastn search against the GenBank NR database was performed, and the top hit with e-value $\leq 10^{-5}$ was reported (Additional file 2: Dataset 1: Whole_transcriptome_expression_TMM_TPM_normalised_filtered_PLTG).

\section{Expression analysis}

Initial reads after quality control were separately aligned back to the $P$. palmivora de novo transcriptome assembly and $N$. benthamiana reference transcriptome. Alignmentbased transcript quantification was done using RSEM (vRSEM-1.2.25, (http://deweylab.github.io/RSEM/) [120]. For $P$. palmivora quantification was performed on the 'Trinity gene' level. For within-sample normalisation TPMs were calculated. Between-sample normalisation was done using the trimmed means (TMM) approach [121]. TMMnormalised TPMs were reported for both P. palmivora and $N$. benthamiana. PCA was performed on the logtransformed TPM values and visualised in R with the help of the 'ggplot2' [122] and 'pheatmap' [123] packages. Overlap between groups of genes identified in the PCA analysis was visualised with the 'Vennerable' package [124]. Differentially expressed genes were identified with the 'edgeR' Bioconductor package [121] following pairwise comparisons between all the samples. The dispersion parameter was estimated from the data with the estimateDisp function on reduced datasets: for P. palmivora we combined close time points (based on PCA) and treated them as pseudoreplicates; for $N$. benthamiana common dispersion was estimated based on 6 uninfected plant samples, treating them as replicates. The resulting common dispersion values of
0.15 and 0.1 were used for $P$. palmivora and $N$. benthamiana analysis, respectively. Most differentially expressed genes ( $\log 2$ (fold change) $\geq 2$ and $p$ value $\leq 10^{-3}$ ) were used to perform hierarchical clustering of samples. Heatmaps for the most differentially expressed genes were generated using the R 'cluster' [125], 'Biobase' [126] and 'qvalue' packages. For the final heatmaps TPMs were log2-transformed and then median-centred by transcript. Plant samples were centred according to the full set of mock and infected samples. Temporal clustering of expression profiles was done with fuzzy clustering ('Mfuzz' Bioconductor package) [69] to adopt gradual temporal changes of gene expression in the course of infection. GO enrichment analysis was done with the help of the 'topGO' Bioconductor package [127]. Gene universe was defined based on $N$. benthamiana genes having expression evidence in our dataset (having TPM $\geq 1$ in at least 3 samples). For the enrichment analysis the exact Fisher test was used, and GO terms with $p$ values $\leq 0.05$ were reported. ReviGO [128] was used to summarise the resulting significant $\mathrm{GO}$ terms and reduce redundancy.

\section{Additional files}

Additional file 1: Table S1. Sequencing and mapping statistics in RNA-seq samples containing P. palmivora (XLS $8 \mathrm{~kb})$ (XLS $8 \mathrm{~kb}$ )

Additional file 2: Dataset 1. P. palmivora de novo transcriptome assembly. Assembly was performed using Trinity 2.1.1 software. Coding sequences (CDS) and corresponding mRNA and amino acid sequences were predicted using TransDecoder with additional homology-based filters. Tar archive contains four files: LILI_transcriptome_v5_converted.fasta - final version of P. palmivora transcriptome; LILI_transcriptome_v5.transdecoder.cds.fasta Transdecoder-predicted CDS; LILI_transcriptome_v5.transdecoder.mRNA.fasta mRNAs for predicted CDS; LILI_transcriptome_v5.transdecoder.pep.fasta amino acid sequences. (GZ $36982 \mathrm{~kb}$ )

Additional file 3: Figure S1. Benchmarking universal single-copy orthologues (BUSCO) genes missing from available Phytophthora genomes and transcriptomes. Figure S2. Amino acid sequence alignment of PLTG_13552 and P. infestans AVR3a ${ }^{\mathrm{EM}}$. Figure S3. HxGPCExxxDD-containing P. palmivora secreted proteins. Figure $\mathbf{S 4}$. Number of differentially expressed genes (DEGs) between infection time points. Figure S5. Validation of dynamic behaviour of P. palmivora DEGs by qRT-PCR. Figure S6. PCR detection of REX1-4 effectors in P. palmivora isolates. Figure S7. Amino acid sequence logos for REX1-4 effectors. Figure S8. Habitus of $N$. benthamiana transgenics used in this study. Figure S9. Subcellular localisation of GFP:REX1-4 proteins in N. benthamiana leaves. Figure S10. Structure of pTrafficLights construct and secretion inhibition assays. Figure S11. Validation of $\mathrm{N}$. benthamiana DEGs by qRT-PCR. Figure S12. Amino acid sequence alignment of TIPTOP and similar N. benthamiana sequences with A. thaliana prePIPL1, prePIP1 and prePIP2. Figure S13. Induction of TIPTOP promoter in response to biotic and abiotic stresses. (PDF $17445 \mathrm{~kb})$

Additional file 4: Table S2. BUSCO genes missing from genomes and transcriptomes of Phytophthora genus. BUSCO genes were annotated using InterProScan based on sequences emitted from hidden Markov model (HMM) profiles. (XLS $35 \mathrm{~kb}$ )

Additional file 5: Dataset 2. N. benthamiana expression table. Raw counts were normalised within and between samples. TMM-normalised TPMs were reported. Functional annotation provided with 1.01 version of N. benthamiana genome was used: (ftp://ftp.solgenomics.net/genomes/ Nicotiana_benthamiana/annotation/Niben101/). (XLSX 3227 kb) 
Additional file 6: Dataset 3. P. palmivora expression table. Raw counts were normalised within and between samples. TMM-normalised TPMs were reported. Not manually curated high throughput annotation (as described Methods) is provided. (XLSX $3138 \mathrm{~kb}$ )

Additional file 7: Dataset 4. Gene ontology (GO) enrichment for $\mathrm{N}$. benthamiana genes up- and downregulated during P. palmivora infection. GO enrichment was done with 'topGO' Bioconductor package. Classic Fisher test was used, only GO terms with $p$ value $<0.05$ reported. (XLSX $24 \mathrm{~kb})$

Additional file 8: Dataset 5. Manually curated P. palmivora secretome. (XLSX $844 \mathrm{~kb})$

Additional file 9: Table S4. P. palmivora isolates. (XLS 8 kb)

Additional file 10: Table S5. PlantPAN analysis of TIPTOP promoter sequence. (XLSX $147 \mathrm{~kb})$

Additional file 11 Table S3. Primers used in this study. (XLS $11 \mathrm{~kb}$ )

\section{Abbreviations}

aa: Amino acid; BUSCO: Benchmarking universal single-copy orthologues; CRN: Crinkler effector; dai: Days after inoculation; DEG: Differentially expressed gene; FDR: False discovery rate; hai: Hours after inoculation; LFC: Log fold change; NPP: Necrosis-inducing Phytophthora protein; ORF: Open reading frame; PCA: Principal component analysis; PI: Propidium iodide, Protease inhibitor; PLTG: Phytophthora palmivora transcribed gene; REX: Putative RXLR-effector expressed; SCR: Small cysteine-rich peptides; SES: Symptom extent stage; SP: Signal peptide; TM: Transmembrane domain; TMM: Trimmed mean of $m$ values; TPM: Transcripts per million

\section{Acknowledgements}

We acknowledge the experimental and annotation help from Abhishek Chatterjee and Schornack lab. We would like to thank Mike Coffey and Joe Win for providing the pathogen isolates and list of oomycete RXLRs. We are indebted to Diane Saunders, Liliana Cano, Jodie Pike and Sophien Kamoun for generating leaf transcriptome sequences. We would like to thank Ruth Le Fevre and Stuart Fawke for proofreading the manuscript.

\section{Funding}

This work was supported by the Gatsby Charitable Foundation (RG62472), by the Royal Society (RG69135) and by the European Research Council (ERC-2014-STG, H2020, 637537).

\section{Availability of data and materials}

The raw fastq files are available in the Sequence Read Archive (SRA) [SRA:SRP096022].

\section{Authors' contributions}

$\mathrm{KF}, \mathrm{CQ}$ and MD generated constructs. TY and EE generated N. benthamiana transgenics. FT and EE characterised transgenics. MD, EE and TH obtained microscopic data. AG performed bioinformatics analysis. EE, AG and SS analysed the data and wrote the manuscript. All authors read and approved the final manuscript.

\section{Competing interests}

The authors declare that they have no competing interests.

\section{Consent for publication}

Not applicable.

\section{Ethics approval and consent to participate} Not applicable.

\section{Publisher's Note}

Springer Nature remains neutral with regard to jurisdictional claims in published maps and institutional affiliations.

\section{Author details}

'Sainsbury Laboratory Cambridge University (SLCU), Cambridge, UK. ${ }^{2}$ Present address: Université Libre de Bruxelles, Bruxelles, Belgium. ${ }^{3}$ Present address: École Normale Supérieure de Lyon, Lyon, France.
Received: 7 April 2017 Accepted: 24 April 2017

Published online: 11 May 2017

\section{References}

1. Erwin DD, Ribeiro OK. Phytophthora diseases worldwide. St. Paul: American Phytopathological Society (APS Press); 1996.

2. Yoshida K, Schuenemann VJ, Cano LM, Pais M, Mishra B, Sharma R, et al. The rise and fall of the Phytophthora infestans lineage that triggered the Irish potato famine. Elife. 2013;2013:1-25.

3. MCHau GRA, Coffey MD. Isozyme diversity in Phytophthora palmivora: evidence for a southeast Asian centre of origin. Mycol Res. 1994;98:1035-43.

4. Scott P, Burgess T, Hardy G. Globalization and Phytophthora. In: Lamour K, editor. Phytophthora: a global perspective. Wallingford: CABl; 2013. p. 226-32.

5. Drenth A, Sendall B. Economic impact of Phytophthora diseases in Southeast Asia. In: Drenth A, Guest DI, editors. Diversity and management of Phytophthora in Southeast Asia. Canberra: Australian Centre for International Agricultural Research (ACIAR); 2004. p. 10-28.

6. Sankar MS, Nath VS, Misra RS, Lajapathy JM. Incidence and identification of Cassava tuber rot caused by Phytophthora palmivora. Arch Phytopathol Plant Prot. 2013:46:741-6.

7. Brooks F. Phytophthora palmivora. Most. 2005;4:2.

8. Torres GA, Sarria GA, Martinez G, Varon F, Drenth A, Guest DI. Bud rot caused by Phytophthora palmivora: a destructive emerging disease of oil palm. Phytopathology. 2016;106:320-9. doi:10.1094/PHYTO-09-15-0243-RVW.

9. Rey T, Chatterjee A, Buttay M, Toulotte J, Schornack S. Medicago truncatula symbiosis mutants affected in the interaction with a biotrophic root pathogen. New Phytol. 2015;206:497-500.

10. Le Fevre R, O'Boyle B, Moscou MJ, Schornack S. Colonization of barley by the broad-host hemibiotrophic pathogen Phytophthora palmivora uncovers a leaf development dependent involvement of MLO. Mol Plant-Microbe Interact. 2016;29:385-95.

11. Daniel R, Guest D. Defence responses induced by potassium phosphonate in Phytophthora palmivora-challenged Arabidopsis thaliana. Physiol Mol Plant Pathol. 2006;67:194-201.

12. Judelson HS, Blanco FA. The spores of Phytophthora: weapons of the plant destroyer. Nat Rev Microbiol. 2005;3:47-58.

13. Petre B, Kamoun S. How do filamentous pathogens deliver effector proteins into plant cells? PLoS Biol. 2014;12, e1001801.

14. Hardham AR. Cell biology of plant-oomycete interactions. Cell Microbiol. 2007:9:31-9

15. Randall TA, Dwyer RA, Huitema E, Beyer K, Cvitanich C, Kelkar H, et al. Largescale gene discovery in the oomycete Phytophthora infestans reveals likely components of phytopathogenicity shared with true fungi. Mol PlantMicrobe Interact. 2005;18:229-43.

16. Raffaele S, Farrer RA, Cano LM, Studholme DJ, MacLean D, Thines M, et al. Genome evolution following host jumps in the Irish potato famine pathogen lineage. Science. 2010;330:1540-3.

17. Whisson SC, Boevink PC, Moleleki L, Avrova AO, Morales JG, Gilroy EM, et al. A translocation signal for delivery of oomycete effector proteins into host plant cells. Nature. 2007:450:115-8.

18. Schornack S, van Damme M, Bozkurt TO, Cano LM, Smoker M, Thines M, et al. Ancient class of translocated oomycete effectors targets the host nucleus. Proc Natl Acad Sci U S A. 2010;107:17421-6.

19. Torto TA, Li S, Styer A, Huitema E, Testa A, Gow NAR, et al. EST mining and functional expression assays identify extracellular effector proteins from the plant pathogen Phytophthora. Genome Res. 2003:13:1675-85.

20. Anderson RG, Deb D, Fedkenheuer K, Mcdowell JM. Recent progress in RXLR effector research. Mol Plant-Microbe Interact. 2015;28:1063-72.

21. Dangl $\mathrm{L}$, Horvath DM, Staskawicz BJ. Pivoting the plant immune system from dissection to deployment. Science. 2013;341:746-51.

22. Vleeshouwers VG, Raffaele S, Vossen JH, Champouret N, Oliva R, Segretin $\mathrm{ME}$, et al. Understanding and exploiting late blight resistance in the age of effectors. Annu Rev Phytopathol. 2011:49:507-31.

23. Yang H, Tao Y, Zheng Z, Li C, Sweetingham MW, Howieson JG. Application of next-generation sequencing for rapid marker development in molecular plant breeding: a case study on anthracnose disease resistance in Lupinus angustifolius L. BMC Genomics. 2012;13:318.

24. Anderson RG, Casady MS, Fee RA, Vaughan MM, Deb D, Fedkenheuer K, et al. Homologous RXLR effectors from Hyaloperonospora arabidopsidis and Phytophthora sojae suppress immunity in distantly related plants. Plant J. 2012;72:1-12. 
25. Cooke DEL, Cano LM, Raffaele S, Bain RA, Cooke LR, Etherington GJ, et al. Genome analyses of an aggressive and invasive lineage of the Irish potato famine pathogen. PLoS Pathog. 2012;8, e1002940.

26. Jones JDG, Dangl JL. The plant immune system. Nature. 2006;444:323-9.

27. Nürnberger T, Nennstiel D, Jabs T, Sacks WR, Hahlbrock K, Scheel D. High affinity binding of a fungal oligopeptide elicitor to parsley plasma membranes triggers multiple defense responses. Cell. 1994;V78:449-60.

28. Brunner F, Rosahl S, Lee J, Rudd JJ, Geiler C, Kauppinen S, et al. Pep-13, a plant defense-inducing pathogen-associated pattern from Phytophthora transglutaminases. EMBO J. 2002;21:6681-8.

29. Ryan CA, Pearce G. Systemins: a functionally defined family of peptide signals that regulate defensive genes in Solanaceae species. Proc Natl Acad Sci U S A. 2003;100:14577-80.

30. Matsubayashi Y. Post-translational modifications in secreted peptide hormones in plants. Plant Cell Physiol. 2011;52:5-13.

31. Boller T, Flury P. Peptides as danger signals: MAMPs and DAMPs. In: Irving H, Gehring C, editors. Plant signaling peptides. Heidelberg: Springer; 2012. p. 163-81.

32. Hou $S$, Wang $X$, Chen $D$, Yang $X$, Wang $M$, Turrà $D$, et al. The secreted peptide PIP1 amplifies immunity through receptor-like kinase 7. PLoS Pathog. 2014;10, e1004331

33. Tai TH, Dahlbeck D, Clark ET, Gajiwala P, Pasion R, Whalen MC, et al. Expression of the Bs2 pepper gene confers resistance to bacterial spot disease in tomato. Proc Natl Acad Sci. 1999;96:14153-8.

34. Zipfel C, Kunze G, Chinchilla D, Caniard A, Jones JDG, Boller T, et al. Perception of the bacterial PAMP EF-Tu by the receptor EFR restricts Agrobacterium-mediated transformation. Cell. 2006;125:749-60.

35. Takemoto D, Jones DA, Hardham AR. GFP-tagging of cell components reveals the dynamics of subcellular re-organization in response to infection of Arabidopsis by oomycete pathogens. Plant J. 2003;33:775-92.

36. Takemoto D. The cytoskeleton as a regulator and target of biotic interactions in plants. Plant Physiol. 2004;136:3864-76.

37. Lipka V, Dittgen J, Bednarek P, Bhat R, Wiermer M, Stein M, et al. Pre- and postinvasion defenses both contribute to nonhost resistance in Arabidopsis. Science. 2005;310:1180-3.

38. Kwon C, Bednarek P, Schulze-Lefert P. Secretory pathways in plant immune response. Plant Physiol. 2008;147:1575-83.

39. An Q, Hückelhoven $\mathrm{R}$, Kogel $\mathrm{KH}$, van Bel AJE. Multivesicular bodies participate in a cell wall-associated defence response in barley leaves attacked by the pathogenic powdery mildew fungus. Cell Microbiol. 2006;8:1009-19.

40. An Q, Ehlers K, Kogel KH, Van Bel AJE, Hückelhoven R. Multivesicular compartments proliferate in susceptible and resistant MLA12-barley leaves in response to infection by the biotrophic powdery mildew fungus. New Phytol. 2006;172:563-76.

41. Lu YJ, Schornack S, Spallek T, Geldner N, Chory J, Schellmann S, et al. Patterns of plant subcellular responses to successful oomycete infections reveal differences in host cell reprogramming and endocytic trafficking. Cell Microbiol. 2012;14:682-97.

42. Inada N, Betsuyaku S, Shimada TL, Ebine K, Ito E, Kutsuna N, et al. Modulation of plant RAB GTPase-mediated membrane trafficking pathway at the interface between plants and obligate biotrophic pathogens. Plant Cell Physiol. 2016:57:1854-64

43. Rose JK, Ham KS, Darvill AG, Albersheim P. Molecular cloning and characterization of glucanase inhibitor proteins: coevolution of a counterdefense mechanism by plant pathogens. Plant Cell. 2002;14:1329-45.

44. Tian M, Huitema E, Da Cunha L, Torto-Alalibo T, Kamoun S. A Kazal-like extracellular serine protease inhibitor from Phytophthora infestans targets the tomato pathogenesis-related protease P69B. J Biol Chem. 2004;279: 26370-7.

45. Tian M, Benedetti B, Kamoun S. A second Kazal-like protease inhibitor from Phytophthora infestans inhibits and interacts with the apoplastic pathogenesisrelated protease P69B of tomato. Plant Physiol. 2005;138:1785-93.

46. Tian M, Win J, Song J, van der Hoorn RAL, van der Knaap E, Kamoun S. A Phytophthora infestans cystatin-like protein targets a novel tomato papainlike apoplastic protease. Plant Physiol. 2007;143:364-77.

47. Song J, Win J, Tian M, Schornack S, Kaschani F, llyas M, et al. Apoplastic effectors secreted by two unrelated eukaryotic plant pathogens target the tomato defense protease Rcr3. Proc Natl Acad Sci U S A. 2009;106:1654-9.

48. Kaschani F, Shabab M, Bozkurt TO, Shindo T, Schornack S, Gu C, et al. An effector-targeted protease contributes to defense against Phytophthora infestans and is under diversifying selection in natural hosts. Plant Physiol. 2010;154:1794-804.

49. Bozkurt TO, Schornack S, Win J, Shindo T, llyas M, Oliva R, et al. Phytophthora infestans effector AVRblb2 prevents secretion of a plant immune protease at the haustorial interface. Proc Natl Acad Sci. 2011;108: 20832-7.

50. Goodin MM, Zaitlin D, Naidu RA, Lommel SA. Nicotiana benthamiana: its history and future as a model for plant-pathogen interactions. Mol Plant Microbe Interact. 2008;21:1015-26.

51. Avrova AO, Boevink PC, Young V, Grenville-Briggs LJ, Van West P, Birch PRJ, et al. A novel Phytophthora infestans haustorium-specific membrane protein is required for infection of potato. Cell Microbiol. 2008;10:2271-84.

52. Ah Fong AMV, Judelson HS. Cell cycle regulator Cdc14 is expressed during sporulation but not hyphal growth in the fungus-like oomycete Phytophthora infestans. Mol Microbiol. 2003;50:487-94.

53. Nakasugi K, Crowhurst RN, Bally J, Wood CC, Hellens RP, Waterhouse PM. De novo transcriptome sequence assembly and analysis of RNA silencing genes of Nicotiana benthamiana. PLoS One. 2013;8, e59534.

54. Haas BJ. Assessing the read content of the transcriptome assembly. 2016. https://github.com/trinityrnaseq/trinityrnaseq/wiki/RNA-Seq-ReadRepresentation-by-Trinity-Assembly.

55. Simão FA, Waterhouse RM, loannidis P, Kriventseva EV, Zdobnov EM. BUSCO: assessing genome assembly and annotation completeness with single-copy orthologs. Bioinformatics. 2015;31:3210-2.

56. Meijer HJG, Mancuso FM, Espadas G, Seidl MF, Chiva C, Govers F, et al. Profiling the secretome and extracellular proteome of the potato late blight pathogen Phytophthora infestans. Mol Cell Proteomics. 2014. doi:10.1074/mcp.M113.035873.

57. Haas BJ, Papanicolaou A, Yassour M, Grabherr M, Blood PD, Bowden J, et al. De novo transcript sequence reconstruction from RNA-seq using the Trinity platform for reference generation and analysis. Nat Protoc. 2013;8:1494-512.

58. Nielsen $\mathrm{H}$, Krogh A. Prediction of signal peptides and signal anchors by a hidden Markov model. Intell Syst Mol Biol. 1998;6:122-30.

59. Bendtsen JD, Nielsen H, Von Heijne G, Brunak S. Improved prediction of signal peptides: SignalP 3.0. J Mol Biol. 2004;340:783-95.

60. Emanuelsson O, Brunak S, von Heijne G, Nielsen H. Locating proteins in the cell using TargetP, SignalP and related tools. Nat Protoc. 2007;2:953-71.

61. Raffaele S, Win J, Cano LM, Kamoun S. Analyses of genome architecture and gene expression reveal novel candidate virulence factors in the secretome of Phytophthora infestans. BMC Genomics. 2010;11:637.

62. Sperschneider J, Williams AH, Hane JK, Singh KB, Taylor JM. Evaluation of secretion prediction highlights differing approaches needed for oomycete and fungal effectors. Front Plant Sci. 2015;6:1-14.

63. Bos JIB, Chaparro-Garcia A, Quesada-Ocampo LM, McSpadden Gardener BB, Kamoun S. Distinct amino acids of the Phytophthora infestans effector AVR3a condition activation of R3a hypersensitivity and suppression of cell death. Mol Plant-Microbe Interact. 2009;22:269-81.

64. Zhang D, Burroughs AM, Vidal ND, lyer LM, Aravind L. Transposons to toxins: the provenance, architecture and diversification of a widespread class of eukaryotic effectors. Nucleic Acids Res. 2016;44:3513-33.

65. van Damme M, Bozkurt TO, Cakir C, Schornack S, Sklenar J, Jones AME, et al. The Irish potato famine pathogen Phytophthora infestans translocates the CRN8 kinase into host plant cells. PLoS Pathog. 2012;8, e1002875.

66. Larroque $M$, Barriot R, Bottin A, Barre A, Rougé P, Dumas B, et al. The unique architecture and function of cellulose-interacting proteins in oomycetes revealed by genomic and structural analyses. BMC Genomics. 2012;13:605.

67. Böhm H, Albert I, Oome S, Raaymakers TM, Van den Ackerveken G, Nürnberger T. A conserved peptide pattern from a widespread microbial virulence factor triggers pattern-induced immunity in Arabidopsis. PLoS Pathog. 2014;10.

68. Hemsworth GR, Henrissat B, Davies GJ, Walton PH. Discovery and characterization of a new family of lytic polysaccharide monooxygenases. Nat Chem Biol. 2013;10:122-6.

69. Kumar L, Futschik ME. Mfuzz: a software package for soft clustering of microarray data. Bioinformation. 2007;2:5-7.

70. Bartetzko V, Sonnewald S, Vogel F, Hartner K, Stadler R, Hammes UZ, et al. The Xanthomonas campestris pv. vesicatoria type III effector protein XopJ inhibits protein secretion: evidence for interference with cell wall-associated defense responses. Mol Plant Microbe Interact. 2009;22:655-64.

71. Chaparro-Garcia A, Wilkinson RC, Gimenez-lbanez S, Findlay K, Coffey MD, Zipfel $C$, et al. The receptor-like kinase SERK3/BAK1 is required for basal resistance against the late blight pathogen Phytophthora infestans in Nicotiana benthamiana. PLoS One. 2011;6:1-10. 
72. Chow CN, Zheng HQ, Wu NY, Chien CH, Da Huang H, Lee TY, et al. PlantPAN 2.0: an update of Plant Promoter Analysis Navigator for reconstructing transcriptional regulatory networks in plants. Nucleic Acids Res. 2016:44:D1154-64.

73. Westermann AJ, Gorski SA, Vogel J. Dual RNA-seq of pathogen and host. Nat Rev Microbiol. 2012;10:618-30.

74. Enguita F, Costa M, Fusco-Almeida A, Mendes-Giannini M, Leitão A. Transcriptomic crosstalk between fungal invasive pathogens and their host cells: opportunities and challenges for next-generation sequencing methods. J Fungi. 2016;2:7.

75. Parra G, Bradnam K, Korf I. CEGMA: a pipeline to accurately annotate core genes in eukaryotic genomes. Bioinformatics. 2007;23:1061-7.

76. Ye W. Sequencing of the litchi downy blight pathogen reveals it is a Phytophthora species with downy mildew-like characteristics. Mol PlantMicrobe Interact. 2015;29:573-83.

77. Hayden KJ, Garbelotto M, Knaus BJ, Cronn RC, Rai H, Wright JW. Dual RNAseq of the plant pathogen Phytophthora ramorum and its tanoak host. Tree Genet Genomes. 2014;10:489-502.

78. Meyer FE, Shuey LS, Naidoo S, Mamni T, Berger DK, Myburg AA, et al. Dual RNA-sequencing of Eucalyptus nitens during Phytophthora cinnamomi challenge reveals pathogen and host factors influencing compatibility. Front Plant Sci. 2016;7:191

79. O'Connell RJ, Thon MR, Hacquard S, Amyotte SG, Kleemann J, Torres MF, et al. Lifestyle transitions in plant pathogenic Colletotrichum fungi deciphered by genome and transcriptome analyses. Nat Genet. 2012;44:1060-5.

80. Attard A, Evangelisti E, Kebdani-Minet N, Panabières F, Deleury E, Maggio C, et al. Transcriptome dynamics of Arabidopsis thaliana root penetration by the oomycete pathogen Phytophthora parasitica. BMC Genomics. 2014;15:538.

81. Ye W, Wang X, Tao K, Lu Y, Dai T, Dong S, et al. Digital gene expression profiling of the Phytophthora sojae transcriptome. Mol Plant-Microbe Interact. 2011:24:1530-9.

82. Windram O, Madhou P, McHattie S, Hill C, Hickman R, Cooke E, et al. Arabidopsis defense against Botrytis cinerea: chronology and regulation deciphered by high-resolution temporal transcriptomic analysis. Plant Cell. 2012;24:3530-57.

83. Shibata Y, Kawakita K, Takemoto D. Age-related resistance of Nicotiana benthamiana against hemibiotrophic pathogen Phytophthora infestans requires both ethylene- and salicylic acid-mediated signaling pathways. Mol Plant-Microbe Interact. 2010;23:1130-42.

84. Eshraghi L, Anderson JP, Aryamanesh N, McComb JA, Shearer B, Hardy GSJE. Suppression of the auxin response pathway enhances susceptibility to Phytophthora cinnamomi while phosphite-mediated resistance stimulates the auxin signalling pathway. BMC Plant Biol. 2014;14:68.

85. Huang H, Qi SD, Qi F, Wu CA, Yang GD, Zheng CC. NtKTI1, a Kunitz trypsin inhibitor with antifungal activity from Nicotiana tabacum, plays an important role in tobacco's defense response. FEBS J. 2010;277:4076-88.

86. Vega K, Kalkum M. Chitin, chitinase responses, and invasive fungal infections. Int J Microbiol. 2012;2012:920459.

87. Li J, Brader G, Palva ET. Kunitz trypsin inhibitor: an antagonist of cell death triggered by phytopathogens and fumonisin B1 in Arabidopsis. Mol Plant. 2008;1:482-95.

88. Shadle GL, Wesley SV, Korth KL, Chen F, Lamb C, Dixon RA. Phenylpropanoid compounds and disease resistance in transgenic tobacco with altered expression of L-phenylalanine ammonia-lyase. Phytochemistry. 2003:64:153-61.

89. Miedes E, Vanholme R, Boerjan W, Molina A. The role of the secondary cell wall in plant resistance to pathogens. Front Plant Sci. 2014;5:358.

90. Tayeh C, Randoux B, Vincent D, Bourdon N, Reignault P. Exogenous trehalose induces defenses in wheat before and during a biotic stress caused by powdery mildew. Phytopathology. 2014;104:293-305.

91. Luo Y, Li WM, Wang W. Trehalose: protector of antioxidant enzymes or reactive oxygen species scavenger under heat stress? Environ Exp Bot. 2008;63:378-84.

92. Chávez-Moctezuma MP, Lozoya-Gloria E. Biosynthesis of the sesquiterpenic phytoalexin capsidiol in elicited root cultures of chili pepper (Capsicum annuum). Plant Cell Rep. 1996;15:360-6.

93. Egea C, Alcázar MD, Candela ME. Capsidiol: its role in the resistance of Capsicum annuum to Phytophthora capsici. Physiol Plant. 1996;98:737-42.

94. Ingram J, Bartels D. The molecular basis of dehydration tolerance in plants. Annu Rev Plant Physiol Plant Mol Biol. 1996;47:377-403.

95. Candat A, Paszkiewicz G, Neveu M, Gautier R, Logan DC, Avelange-Machere $\mathrm{M}-\mathrm{H}$, et al. The ubiquitous distribution of late embryogenesis abundant proteins across cell compartments in Arabidopsis offers tailored protection against abiotic stress. Plant Cell. 2014;26:3148-66.
96. Serrazina S, Santos C, Machado H, Pesquita C, Vicentini R, Pais MS, et al. Castanea root transcriptome in response to Phytophthora cinnamomi challenge. Tree Genet Genomes. 2015;11:1-19.

97. Shen D, Chai C, Ma L, Zhang M, Dou D. Comparative RNA-Seq analysis of Nicotiana benthamiana in response to Phytophthora parasitica infection. Plant Growth Regul. 2016;80:59-67.

98. Segretin ME, Pais M, Franceschetti M, Chaparro-Garcia A, Bos JIB, Banfield MJ, et al. Single amino acid mutations in the potato immune receptor R3a expand response to Phytophthora effectors. Mol Plant-Microbe Interact. 2014;27:624-37.

99. Chapman S, Stevens L, Boevink PC, Engelhardt S, Alexander CJ, Harrower B, et al. Detection of the virulent form of AVR3a from Phytophthora infestans following artificial evolution of potato resistance gene R3a. PLoS One. 2014;9, e110158.

100. Albert I, Böhm H, Albert M, Feiler CE, Imkampe J, Wallmeroth N, et al. An RLP23SOBIR1-BAK1 complex mediates NLP-triggered immunity. Nat Plants. 2015;1:15140.

101. Bozkurt TO, Belhaj K, Dagdas YF, Chaparro-Garcia A, Wu CH, Cano LM, et al. Rerouting of plant late endocytic trafficking toward a pathogen interface. Traffic. 2015;16:204-26.

102. Reeksting BJ, Olivier NA, van den Berg N. Transcriptome responses of an ungrafted Phytophthora root rot tolerant avocado (Persea americana) rootstock to flooding and Phytophthora cinnamomi. BMC Plant Biol. 2016;16:205.

103. Simon $R$, Dresselhaus T. Peptides take centre stage in plant signalling. J Exp Bot. 2015;66:5135-8.

104. Vie AK, Najafi J, Liu B, Winge P, Butenko MA, Hornslien KS, et al. The IDA IDA-LIKE and PIP/PIP-LIKE gene families in Arabidopsis: phylogenetic relationship, expression patterns, and transcriptional effect of the PIPL3 peptide. J Exp Bot. 2015;66:5351-65.

105. Attard A, Gourgues M, Callemeyn-Torre N, Keller H. The immediate activation of defense responses in Arabidopsis roots is not sufficient to prevent Phytophthora parasitica infection. New Phytol. 2010;187:449-60.

106. Sokal RR, Rohlf FJ. Biometry: the principles and practices of statistics in biological research. New York: W. H. Freeman; 1995.

107. You FM, Huo N, Gu YQ, Luo M-C, Ma Y, Hane D, et al. BatchPrimer3: a high throughput web application for PCR and sequencing primer design. BMC Bioinformatics. 2008;9:253.

108. Yan HZ, Liou RF. Selection of internal control genes for real-time quantitative RT-PCR assays in the oomycete plant pathogen Phytophthora parasitica. Fungal Genet Biol. 2006;43:430-8.

109. Liu D, Shi L, Han C, Yu J, Li D, Zhang Y. Validation of reference genes for gene expression studies in virus-infected Nicotiana benthamiana using quantitative real-time PCR. PLoS One. 2012;7, e46451.

110. Sparkes IA, Runions J, Kearns A, Hawes C. Rapid, transient expression of fluorescent fusion proteins in tobacco plants and generation of stably transformed plants. Nat Protoc. 2006;1:2019-25.

111. Martin M. Cutadapt removes adapter sequences from high-throughput sequencing reads. EMBnet J. 2011;17:10-2.

112. Kim D, Pertea G, Trapnell C, Pimentel H, Kelley R, Salzberg SL. TopHat2: accurate alignment of transcriptomes in the presence of insertions, deletions and gene fusions. Genome Biol. 2013;14:R36.

113. Emanuelsson O, Nielsen H, Brunak S, von Heijne G. Predicting subcellular localization of proteins based on their $\mathrm{N}$-terminal amino acid sequence. J Mol Biol. 2000;300:1005-16.

114. Krogh A, Larsson B, von Heijne G, Sonnhammer ELL. Predicting transmembrane protein topology with a hidden Markov model: application to complete genomes. J Mol Biol. 2001;305:567-80.

115. Stornaiuolo M. KDEL and KKXX retrieval signals appended to the same reporter protein determine different trafficking between endoplasmic reticulum, intermediate compartment, and Golgi complex. Mol Biol Cell. 2003;14:2372-84.

116. Zdobnov EM, Apweiler R. InterProScan — an integration platform for the signature-recognition methods in InterPro. Bioinformatics. 2001;17:847-8.

117. Nguyen Ba AN, Pogoutse A, Provart N, Moses AM. NLStradamus: a simple hidden Markov model for nuclear localization signal prediction. BMC Bioinformatics. 2009:10:202.

118. Cokol M, Nair R, Rost B. Finding nuclear localization signals. EMBO Rep. 2000;1:411-5.

119. Biasini M, Bienert S, Waterhouse A, Arnold K, Studer G, Schmidt T, et al. SWISS-MODEL: modelling protein tertiary and quaternary structure using evolutionary information. Nucleic Acids Res. 2014;42:252-8.

120. Li B, Dewey CN. RSEM: accurate transcript quantification from RNA-Seq data with or without a reference genome. BMC Bioinformatics. 2011;12:323.

121. Robinson MD, Oshlack A. A scaling normalization method for differential expression analysis of RNA-seq data. Genome Biol. 2010;11:R25.

122. Wickham H. ggplot2: elegant graphics for data analysis. New York: Springer; 2009. 
123. Raivo K. pheatmap: Pretty Heatmaps. 2013. https://cran.r-project.org/web/ packages/pheatmap/index.html.

124. Swinton J. Venn diagrams in R with the Vennerable package. 2013. https:// github.com/ss229/Nennerable.

125. Maechler M, Rousseeuw P, Struyf A, Hubert M, Hornik K. cluster: cluster analysis basics and extensions. R package version 2.0.5. 2016.

126. Huber W, Carey VJ, Gentleman R, Anders S, Carlson M, Carvalho BS, et al. Orchestrating high-throughput genomic analysis with Bioconductor. Nat Methods. 2015;12:115-21.

127. Alexa A, Rahnenfuhrer J. topGO: enrichment analysis for Gene Ontology. 2010. http://www.bioconductor.org/packages/release/bioc/html/topGO. html.

128. Supek F, Bošnjak M, Škunca N, Šmuc T. Revigo summarizes and visualizes long lists of gene ontology terms. PLoS One. 2011;6. https://doi.org/10. 1371/journal.pone.0021800

Submit your next manuscript to BioMed Central and we will help you at every step:

- We accept pre-submission inquiries

- Our selector tool helps you to find the most relevant journal

- We provide round the clock customer support

- Convenient online submission

- Thorough peer review

- Inclusion in PubMed and all major indexing services

- Maximum visibility for your research

Submit your manuscript at www.biomedcentral.com/submit 